\title{
PI3-kinase activation is critical for host barrier permissiveness to Listeria monocytogenes
}

\author{
Grégoire Gessain, ${ }^{1,2,3}$ Yu-Huan Tsai, ${ }^{1,2,3 *}$ Laetitia Travier, ${ }^{1,2 *}$ \\ Matteo Bonazzi, ${ }^{4,5,6}$ Solène Grayo, ${ }^{1,2}$ Pascale Cossart, ${ }^{4,5,6}$ \\ Caroline Charlier, ${ }^{1,2,7,8}$ Olivier Disson, ${ }^{1,2 * *}$ and Marc Lecuit ${ }^{1,2,7,8 * *}$
}

IInstitut Pasteur, Biology of Infection Unit, F-75015 Paris, France

${ }^{2}$ Institut National de la Santé et de la Recherche Médicale, U1117, F-75015 Paris, France

${ }^{3}$ Université Paris Diderot, Sorbonne Paris Cité, Cellule Pasteur, F-75013 Paris, France

${ }^{4}$ Institut Pasteur, Bacteria Cell Interaction Unit, F-75015 Paris, France

5 Institut National de la Santé et de la Recherche Médicale, U604, F-75015 Paris, France

${ }^{6}$ Institut National de la Recherche Agronomique USC2020, F-75015 Paris, France

${ }^{7}$ Institut Pasteur, French National Reference Center and World Health Organization Collaborating Centre on Listeria,

F-75015 Paris, France

${ }^{8}$ Paris Descartes University, Sorbonne Paris Cité, Division of Infectious Diseases and Tropical Medicine, Necker-Enfants

Malades University Hospital, Institut Imagine, F-75015 Paris, France

Invasion of nonphagocytic cells, a critical property of Listeria monocytogenes $(\mathrm{Lm})$ that enables it to cross host barriers, is mediated by the interaction of two bacterial surface proteins, InIA and InIB, with their respective receptors E-cadherin and c-Met. Although InIA-E-cadherin interaction is necessary and sufficient for $\mathrm{Lm}$ crossing of the intestinal barrier, both InIA and InIB are required for $L m$ crossing of the placental barrier. The mechanisms underlying these differences are unknown. Phosphoinositide 3-kinase (PI3-K) is involved in both InIA- and InIB-dependent pathways. Indeed, InIA-dependent entry requires $\mathrm{PI} 3-\mathrm{K}$ activity but does not activate it, whereas InIB-c-Met interaction activates $\mathrm{PI} 3-\mathrm{K}$. We show that $L m$ intestinal target cells exhibit a constitutive PI3-K activity, rendering InIB dispensable for InIA-dependent $L m$ intestinal barrier crossing. In contrast, the placental barrier does not exhibit constitutive PI3-K activity, making InIB necessary for InIA-dependent Lm placental invasion. Here, we provide the molecular explanation for the respective contributions of $\operatorname{InI} \mathrm{A}$ and $\operatorname{InIB}$ to $L m$ host barrier invasion, and reveal the critical role of InIB in rendering cells permissive to InIA-mediated invasion. This study shows that PI3-K activity is critical to host barrier permissiveness to microbes, and that pathogens exploit both similarities and differences of host barriers to disseminate.

\section{CORRESPONDENCE}

Marc Lecuit:

marc.lecuit@pasteur.fr

Abbreviations used: acc-Ecad, accessible Ecad; EC, extruding cell; Ecad, E-cadherin; EEF, enterocyte located in epithelial fold; EGF, epidermal growth factor; esiRNA, endoribonucleaseprepared siRNA; GC, goblet cell; hEcad, human Ecad; HGF, hepatocyte growth factor; IGF, insulin-like growth factor; InlA, internalin A; Lm, Listeria monocytogenes; $L m-$ InlA $^{\mathrm{m}}, \mathrm{Lm}$ expressing a murinized version of InlA; mEcad, mouse Ecad; Ncad, N-cadherin; PI3-K, phosphoinositide 3-kinase WGA, wheat germ agglutinin.
The skin and mucosa delimit a frontier between host internal milieu and the external nonsterile environment. Within the host, the blood-brain and placental barriers provide additional protection to the brain and the fetus, respectively. Among their many physiological roles, these host barriers act as critical ramparts against microbial dissemination, yet several pathogens have selected mechanisms to breach host barriers. In addition to what it teaches us on the biology of

\footnotetext{
*Y.-H. Tsai and L. Travier contributed equally to this paper. **O. Disson and M. Lecuit contributed equally to this paper. M. Bonazzi's present address is Centre National de la Recherche Scientifique, UMR 5236, CPBS, 34293 Montpellier, France.
}

host barriers, the deciphering of host-pathogen interactions that mediate host barriers breaching is key to understand the pathophysiology of human systemic infections. Here, we have used Listeria monocytogenes $(\mathrm{Lm})$ as a model invasive bacteria and discovered a novel molecular mechanism underlying host barrier permissiveness to microbes.

$L m$ is a foodborne pathogen responsible for human listeriosis, a systemic infection with a $30 \%$ mortality rate (Swaminathan and

O 2015 Gessain et al. This article is distributed under the terms of an AttributionNoncommercial-Share Alike-No Mirror Sites license for the first six months after the publication date (see http://www.rupress.org/terms). After six months it is
the available under a Creative Commons License (Attribution-Noncommercial-Share Alike 3.0 Unported license, as described at http://creativecommons.org/licenses/ by-nc-sa/3.0/). 
Gerner-Smidt, 2007). Upon ingestion, Lm can survive and multiply in the intestinal lumen, actively cross the intestinal barrier, and disseminate within the host. In addition, $L m$ can cross the blood-brain barrier and cause meningitis and encephalitis, as well as the placental barrier, resulting in abortion and neonatal infection (Lecuit, 2007).

The ability of $L m$ to cross these host barriers relies on its capacity to invade nonphagocytic cells. This is mediated by two bacterial surface proteins: internalin (InlA) and InlB (Gaillard et al., 1991; Dramsi et al., 1995; Disson and Lecuit, 2013). InlA is a Lm surface protein covalently linked to its cell wall that interacts with E-cadherin (Ecad; Mengaud et al., 1996), a transmembrane protein that forms adherens junctions between epithelial cells. InlA-Ecad interaction is species specific: human Ecad (hEcad) is a receptor for InlA, whereas mouse Ecad (mEcad) is not (Lecuit et al., 1999). InlA mediates the crossing of the intestinal epithelium in species permissive to InlA-Ecad interaction, such as guinea pig, gerbil, humanized mouse models expressing human E-cadherin (hEcad transgenic mice) or a "humanized" version of $\mathrm{mEcad}$ (knock-in mice KIE16P; Lecuit et al., 2001; Disson et al., 2008), and human (Jacquet et al., 2004).

InlB is an $L m$ surface protein noncovalently bound to its cell wall. It interacts with three host cell surface molecules: (1) c-Met, the receptor of hepatocyte growth factor (HGF; Shen et al., 2000), a transmembrane tyrosine kinase protein involved in cell growth, motility, and development; (2) gC1qR, the receptor for the globular head domain of the complement component C1q (Braun et al., 2000); and (3) glycosaminoglycans (Jonquières et al., 2001). Binding of InlB to c-Met mimics HGF signaling and induces membrane ruffling and cell scattering via the activation of the type IA phosphoinositide 3kinase $(\mathrm{PI} 3-\mathrm{K})$ that phosphorylates the plasma membrane lipid second messenger phosphoinositide-4,5-bisphosphate (PIP2) into phosphoinositide-3,4,5-trisphosphate (PIP3). This process is critical for $\mathrm{Lm}$ internalization (Ireton et al., 1996; Jiwani et al., 2012). InlB-dependent entry is also species specific, as the human, gerbil, and mouse c-Met act as receptors for InlB, but the c-Met of guinea pig and rabbit do not (Khelef et al., 2006).

In animal models permissive to both InlA and InlB, these proteins are involved in $\mathrm{Lm}$ crossing of host barriers (Disson et al., 2008). InlA is necessary and sufficient to mediate the crossing of the intestinal barrier by targeting luminally accessible Ecad (acc-Ecad), in particular on mucus-secreting goblet cells (GCs; Lecuit et al., 2001; Nikitas et al., 2011), whereas InlB is not involved in this process (Khelef et al., 2006). In contrast, in third-trimester and term placentas, the crossing of the trophoblastic barrier requires the conjugated action of InlA and InlB (Lecuit et al., 2004; Disson et al., 2008), and $L m$ invades the syncytiotrophoblasts that express Ecad accessible to mother blood-borne bacteria (Lecuit et al., 2004).

Here, we investigated the molecular mechanisms underlying the respective requirements of InlA and InlB in the crossing of the intestinal and placental barriers. The internalization of $L m$ into cells is an active process that requires host protein tyrosine phosphorylation and actin cytoskeleton rearrangements (Cossart et al., 2003). Several growth factors, such as HGF, EGF (epidermal growth factor), insulin, and IGF (insulin-like growth factor) activate PI3-K signaling via their respective tyrosine-kinase transmembrane receptors (Engelman et al., 2006). Acting as an agonist of HGF, the bona fide ligand of c-Met, InlB also triggers PI3-K activation, in contrast to InlA, which does not (Ireton et al., 1996, 1999).

Our working hypothesis is that host basal cell PI3-K signaling, as assessed by PI3-K-dependent phosphorylation of Akt, might have a direct impact on the requirement of InlB in bacterial host barrier invasion. We therefore investigated the level of P-Akt at steady state in Lm-target cells at the intestinal and placental barrier levels. We show that $L m$ intestinal target cells exhibit a constitutive PI3-K activity, rendering InlB dispensable for InlA-dependent $L m$ intestinal barrier crossing. In contrast, the placental barrier does not exhibit constitutive PI3-K activity, making InlB necessary for InlA-dependent Lm placental invasion. This provides the molecular explanation for the respective contributions of InlA and InlB to $\mathrm{Lm}$ host barriers invasion, and reveals the critical role of InlB in rendering cells permissive to InlA-mediated invasion. This study shows that PI3-K activity is critical to host barrier permissiveness to microbes, and that pathogens exploit both similarities and differences of host barriers to disseminate.

\section{RESULTS}

\section{$L m$ intestinal target cells exhibit a higher intrinsic Akt} phosphorylation level than $\mathbf{L m}$-placental target cells in vivo

We investigated the expression of the InlA and InlB receptors (Ecad and c-Met, respectively) in placental and intestinal tissues of uninfected KIE16P mice by immunofluorescence and found that they are both expressed (Fig. 1 A) as previously shown in gerbils (Disson et al., 2008). We assessed the activation status of PI3-K in these tissues by determining the phosphorylation of the serine/threonine kinase Akt (P-Akt), which reflects PI3-K activity upon growth factor signaling (Vanhaesebroeck et al., 2012). Immunostaining of mouse placental and intestinal tissues for P-Akt revealed that it is hardly detectable in the placenta (Fig. 1, B and C). In contrast, it is electively detectable in three specific epithelial cell subsets of the intestine: the mucus-secreting goblet cells (GCs) in which the mucus is stained by wheat germ agglutinin (WGA), the extruding cells (ECs), and the enterocytes located in epithelial folds (EEFs), in contrast to other enterocytes (Fig. 1, $\mathrm{C}$ and $\mathrm{D})$.

Importantly, we have previously shown that GCs, ECs, and EEFs are the cell subsets that express luminally acc-Ecad and are targeted by $L m$ in vivo (Nikitas et al., 2011). We therefore examined the link between PI3-K activity and Ecad accessibility on the intestinal epithelium, particularly in GCs, ECs, and EEFs. We observed by confocal microscopy that both P-Akt and acc-Ecad are detectable in GCs, ECs, and EEFs (Fig. 1 E).

To confirm the specificity of the P-Akt labeling, we preincubated the intestinal tissue with the Ser-473 P-Akt blocking 

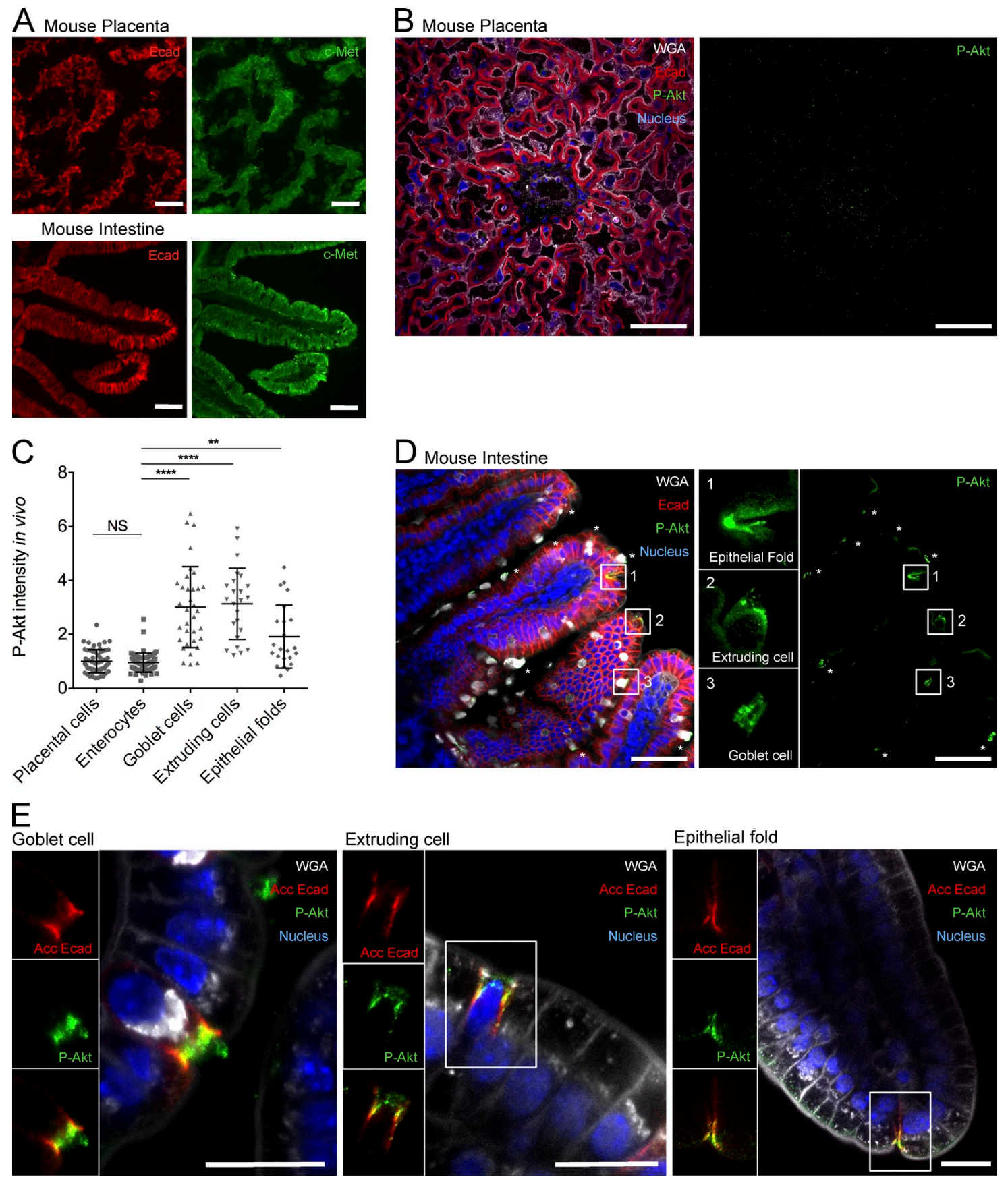

Figure 1. Lm intestinal and placental target cells express InIA and InIB receptors E-cadherin and c-Met. $L m$ intestinal target cells express high Akt phosphorylation level in vivo whereas placental cells do not. (A) Representative wide-field microscopy images of $8 \mu \mathrm{m}$ cryotome sections taken from uninfected KIE16P mouse tissues: placenta (top) and intestine (bottom). Tissues were stained for E-cadherin (red) and c-Met (green). (B) Representative confocal microscopy images of vibratome sections taken from uninfected KIE16P mouse placental tissue stained for WGA (white), E-cadherin (red), P-Akt (green), and Hoechst (blue). (right) P-Akt control staining. (C) Quantification of P-Akt intensity in vivo, in placental cells, enterocytes, GCs, ECs, and EEFs measured from at least 15 fields ( $n=3$ independent KIE16P mice). Each dot corresponds to one quantified cell and at least 25 cells were quantified per cell subset. A one-way ANOVA test followed by a Bonferroni's multiple comparisons was performed. Error bars, SD. Values of P-Akt intensity are expressed as the fold-change of intensity relative to enterocytes, for which the value was normalized to 1. (D) Representative confocal microscopy images of vibratome sections taken from uninfected KIE16P mouse intestinal tissue stained for WGA (white), E-cadherin (red), P-Akt (green) and Hoechst (blue). (right) P-Akt control staining. Asterisks indicate P-Akt positive GCs. Magnification for insets of boxed regions 1, 2, and 3: 200\%. (E) Representative confocal microscopy images of whole-mount intestinal villi sections taken from uninfected KIE16P mice, and stained for luminally accessible E-cadherin (acc-Ecad; red), WGA (white), P-Akt (green), and Hoechst (blue). (left) Separated channels and merge of boxed regions, showing apparent colocalization of acc-Ecad (red) and P-Akt (green) in a GC (left), an EC (middle), and an EEF (right). Microscopy results were representative of at least two independent mice. Bars: (A and E) $20 \mu \mathrm{m}$; (B and D) $50 \mu \mathrm{m}$. 
A

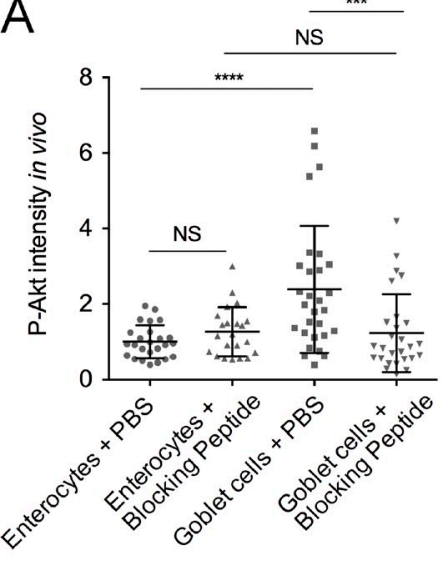

C

Goblet cell

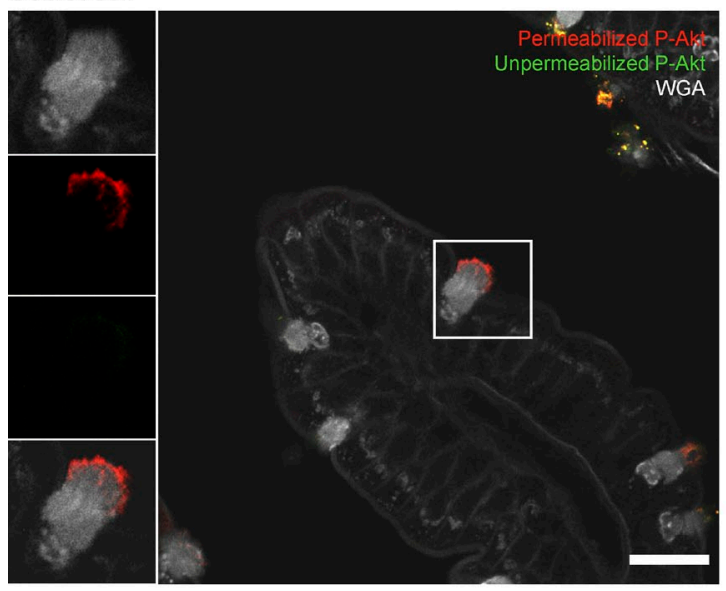

D

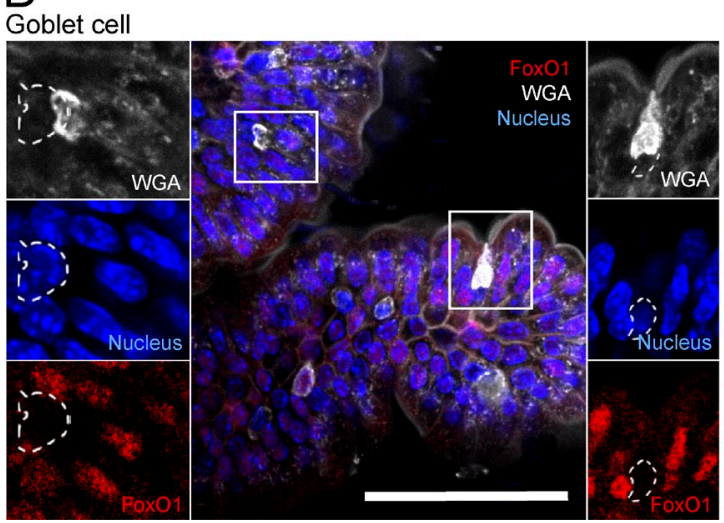

B

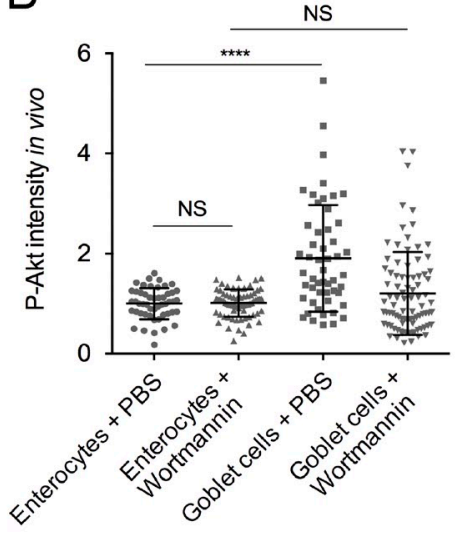

\section{Extruding cell}

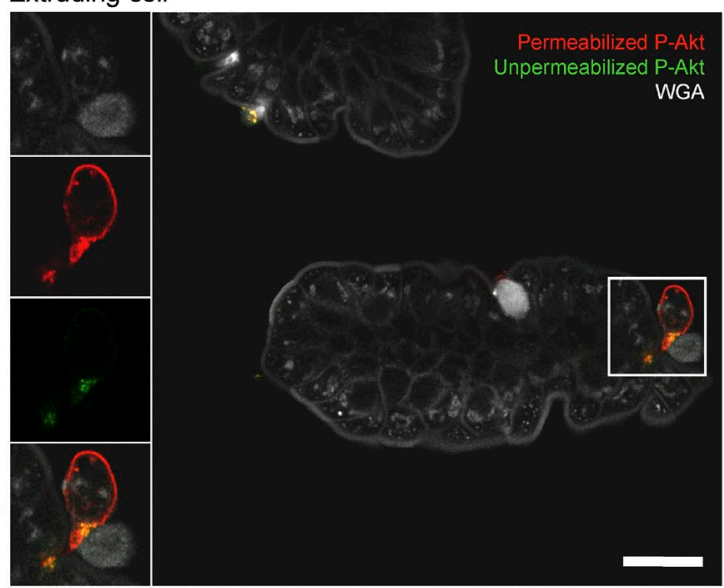

E

Goblet cell

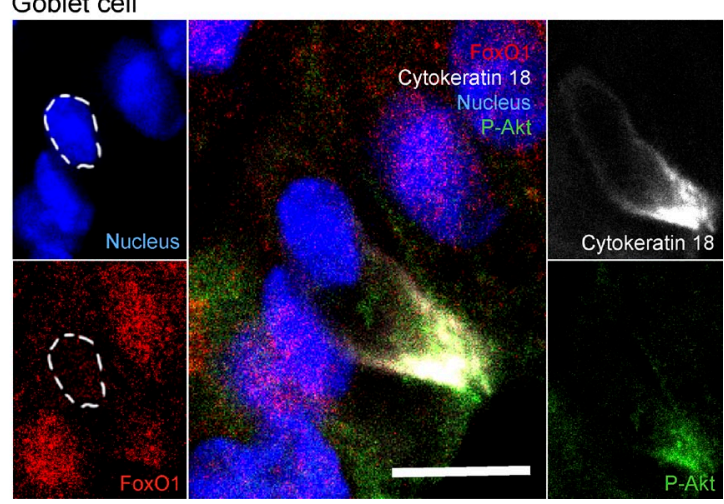

Figure 2. Goblet cells and extruding cells exhibit a specific P-Akt staining, and goblet cells present an extranuclear Fox01 staining in vivo, which reflects the phosphorylation of Akt. (A) Quantification of P-Akt intensity in vivo, in enterocytes and GCs, measured from at least 10 fields ( $n=2$ independent KIE16P mice), where PBS or a Ser-473 P-Akt blocking peptide was added to the intestinal tissue. (B) Quantification of P-Akt intensity in vivo, in enterocytes and GCs, measured from at least 15 fields ( $n=2$ different intestinal ligated loops of 2 independent KIE16P mice), where wortmannin $(10 \mu \mathrm{M})$ was injected for $1 \mathrm{~h}$. Each dot corresponds to one quantified cell and at least 20 cells were quantified per cell subset. A one-way ANOVA test followed by a Bonferroni's multiple comparisons was performed. Error bars, SD. Values of P-Akt intensity are expressed as the fold-change of intensity relative to enterocytes, for which the value was normalized to 1. (C) Representative confocal microscopy images of whole-mount intestinal villi sections take from uninfected KIE16P mice, and stained for P-Akt before the permeabilization step (in green). The tissue was then permeabilized and stained for P-Akt (red) and WGA (white). Thus, extracellular P-Akt was labeled in yellow (apparent colocalization of extracellular P-Akt green and total P-Akt red) and intracellular P-Akt was labeled in red. Red P-Akt was strictly observed at intracellular locations confirming the specificity of the labeling. (left) GC; (right) 
peptide and showed that P-Akt labeling is significantly reduced in GCs (Fig. 2 A). In addition, as Akt may be induced independently from PI3-K signaling (Guo et al., 2011; Mahajan and Mahajan, 2012), we specifically inhibited PI3$\mathrm{K}$ activity to investigate whether the phosphorylation of Akt in GCs is PI3-K dependent. We injected wortmannin, a pharmacological PI3-K inhibitor (Ireton et al., 1996), into intestinal ligated loops of KIE16P mice and observed a significant decrease of P-Akt-positive GCs (Fig. 2 B), demonstrating that the increased level of Akt phosphorylation in GCs as compared with enterocytes is PI3-K dependent. Finally, as intraluminal mucus can lead to false-positive immunostaining, we labeled extracellular and total intestinal P-Akt, and could demonstrate a specific intracellular P-Akt staining in GCs and ECs (Fig. 2 C).

We also assessed FoxO1 intracellular location to confirm the activation status of PI3-K and the specificity of P-Akt staining. FoxO1 is excluded from the nucleus and relocalized in the cytosol for degradation when Akt is phosphorylated (Biggs et al., 1999; Wang et al., 2008). Consistently, FoxO1 was systematically excluded from the nucleus of GCs (Fig. 2 D), confirming Akt phosphorylation and therefore suggesting a constitutive PI3-K activity in these cells. Last, we stained the intestinal epithelium with cytokeratin 18, a marker of GCs (McDole et al., 2012), the major portal of entry of $L m$ at the intestinal level, and confirmed that Akt was phosphorylated in cytokeratin 18-positive cells, and FoxO1 was excluded from the nucleus (Fig. $2 \mathrm{E}$ ).

Together, these results strongly suggest that mouse intestinal epithelial cells expressing acc-Ecad, which are targeted by $L m$, have a constitutively higher basal PI3-K activity than the mouse placental tissue.

\section{Akt phosphorylation level is higher in cultured human intestinal cells than trophoblastic cells}

To investigate the underlying molecular mechanisms of these in vivo observations, we investigated PI3-K activity in uninfected human intestinal LS174T cells, a well-established intestinal mucus secreting GC-like cell line (Tom et al., 1976), and in uninfected human trophoblastic cell line Jar (Pattillo et al., 1971). These two human cell lines express both the InlA and InlB receptors Ecad and c-Met (Fig. 3, A and B), as observed in mice in vivo (Fig. 1 A). By assessing Akt phosphorylation level (Fig. 3, A, C, and D) and FoxO1 cytosolic sequestration (Fig. 3 E), we demonstrated that intestinal LS174T cells exhibit a high constitutive PI3-K activity, whereas placental Jar cells do not. These data suggest that LS174T and Jar cell lines are relevant human in vitro models to study the contribution of PI3-K in Lm intestinal and placental barrier invasion.

\section{Phosphorylation of Akt inversely correlates with InIB-dependent entry in vitro}

To study the impact of PI3-K activity on InlA and InlB requirement for $\mathrm{Lm}$ internalization, we performed gentamicin survival assays in LS174T and Jar cells. As previously shown in cells expressing hEcad (Mengaud et al., 1996; Lecuit et al., 1999), the entry of $L m$ inlA deletion mutant $(\Delta i n l A)$ was reduced compared with WT Lm in both LS174T and Jar cells (Fig. 4, A and B). In contrast, inlB deletion $(\Delta i n l B)$ had no significant effect on Lm entry into LS174T cells, in which there is an elevated basal PI3-K activity, but reduced Lm entry into Jar cells, where the basal PI3-K activity is low (Fig. 4, A and B). Immunoblotting of EGD- and $\Delta$ inlB-infected LS174T and Jar cells revealed that: (i) EGD activates PI3-K; (ii) PI3-K activation is InlB dependent, as $\Delta$ inlB-infected cells have a similar level of Akt phosphorylation compared with PBStreated cells; and (iii) InlB-induced Akt phosphorylation is greater in Jar cells where P-Akt basal level is lower than in LS174T cells where P-Akt basal level is higher (Fig. 4, C and D). These results show that InlB is dispensable for $L m$ entry in LS174T intestinal cells in which PI3-K is constitutively activated, but is required for $\mathrm{Lm}$ invasion of Jar placental cells in which PI3-K is not activated before infection, as previously shown in vivo at the intestinal and placental level, respectively (Disson et al., 2008).

We next confirmed that the entry of Lm was PI3-Kdependent in LS174T and Jar cell lines, as previously shown in the epithelial cell lines Vero and Caco-2 (Ireton et al., 1996). The invasion of WT Lm, and isogenic mutants $\Delta i n l A$ and $\Delta i n l B$, was reduced in the context of a partial knockdown of the type IA PI3-K p $85 \alpha$ subunit by siRNAs in LS174T (Fig. 4, E and F) and Jar cells (Fig. 4, G and H). Of note, the partial inhibition of PI3-K activity also revealed a role for InlB in Lm entry into LS174T cells (Fig. 4 E; see CI-1033 experiments in next paragraph). Furthermore, treatment with the PI3-K irreversible inhibitors LY294002 and wortmannin completely abolished the invasion of WT Lm and isogenic mutants $\Delta i n l A$ and $\Delta i n l B$ (Ireton et al., 1996) in LS174T (Fig. 4, I and K) and Jar cells (Fig. 4, J and L), confirming that Lm entry into these cells is PI3-K dependent. Note that wortmannin and LY294002 had no effect on InlA and InlB expression (Fig. 4 M) and bacterial growth (Fig. $4 \mathrm{~N}$ ).

We next investigated the correlation between PI3-K basal activity and InlB requirement for $L m$ invasion by modulating

EC. Magnification for insets of boxed regions: 50\%. (D) Representative confocal microscopy stack projection of whole-mount intestinal villi take from uninfected KIE16P mice stained for WGA (white), Fox01 (red), and Hoechst (blue). Left and right panels show separated channels of boxed regions, showing the absence of Fox 01 in the nucleus of GCs, and apparent colocalization of Fox01 and Hoechst staining in the neighboring enterocytes. Magnification for insets of boxed regions: 75\%. (E) Representative confocal microscopy images of vibratome sections of intestinal villi taken from uninfected KIE16P mice stained for cytokeratin 18 (white), P-Akt (green), Fox01 (red), and Hoechst (blue). Left and right panels show separated channels, showing a GC positive for cytokeratin 18, where Akt is phosphorylated and Fox01 is excluded from the nucleus. Microscopy results were representative from at least two independent mice. Bars: (C and D) $20 \mu \mathrm{m}$; (E) $10 \mu \mathrm{m}$. 

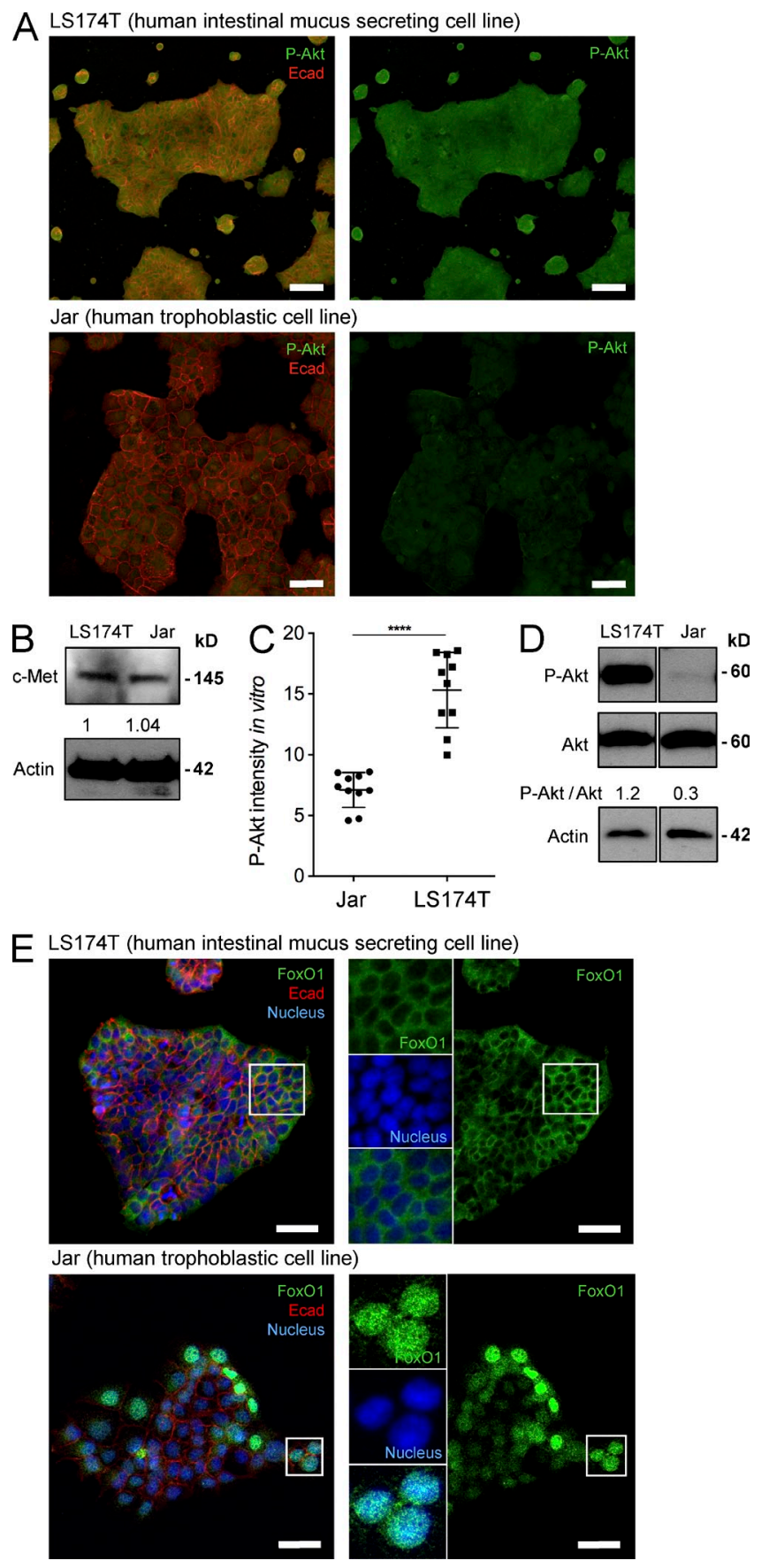

Figure 3. Intestinal LS174T and placental Jar cell lines express InIA and InIB receptors E-cadherin and c-Met, and the LS174T cell line exhibits phosphorylated Akt, whereas Jar cell line does not. (A) Representative confocal microscopy images of LS174T (top) and Jar cells (bottom) stained with E-cadherin (red) and P-Akt (green). (right) P-Akt control staining. (B) Whole-cell extracts from Jar and LS174T cells were immunoblotted for c-Met. Densitometry was performed. (C) Quantification of P-Akt intensity in vitro in Jar and LS174T cells measured from at least 10 fields ( $n=2$ independent experiments). An unpaired Student's $t$ test was performed. Error bars, SD. (D) Whole-cell extracts from Jar and LS174T immunoblotted for P-Akt and total Akt. Densitometry was performed and the ratio of P-Akt over total Akt is relative to the PBS, for which the value is normalized to 1. (E) Representative confocal microscopy the PI3-K-dependent signaling in LS174T and Jar cells and investigating its impact on InlB-requirement for Lm entry. In LS174T cells, EGF-R and IGF-R signaling induce PI3-K activation (Wang et al., 2008). To decrease PI3-K signaling in LS174T cells without affecting HGF-R pathway downstream of InlB-c-Met signaling, we treated cells before infection with the pan-ErbB inhibitor CI-1033 (Yusta et al., 2009), which inhibits EGF-R activation. Addition of CI-1033 dose dependently decreased the amount of P-Akt without significantly affecting the total amount of Akt, indicating that CI-1033 specifically inhibits Akt phosphorylation and thereby PI3-K signaling in LS174T cells (Fig. 5 A). Whereas InlB is not required for $\mathrm{Lm}$ invasion of LS174T cells (Fig. $5 \mathrm{~B}$, black bars), it is in LS174T cells treated with CI-1033 (Fig. 5 B, gray bars), as shown in the previous paragraph siRNA-treated LS174T cells in which the PI3-K activity is partially reduced. Therefore, by down-regulating EGF-dependent PI3-K activation before infection, CI-1033 reveals a role for InlB-c-Met interaction in promoting InlA-dependent entry by activating PI3-K (Fig. 5 C).

In a converse approach, we experimentally induced PI3-K activation in Jar cells before infection, in an attempt to suppress the requirement of InlB in $L m$ entry. First, we treated cells with EGF that activates PI3-K independently of HGF and InlB. Treatment with EGF alone resulted in a strong but transient phosphorylation of Akt (Fig. 5 D). Therefore, by use of endoribonuclease-prepared siRNA (esiRNA), we knocked down PTEN, a phosphatase that dephosphorylates PIP3 into PIP2, to maintain a steady PI3-K signaling (Maehama and Dixon, 1998). PTEN depletion was assessed by immunoblot (Fig. 5 E). The synergistic action of esiRNA and EGF strongly increased the amount of $\mathrm{P}-\mathrm{Akt}$, indicating a steady induction of PI3-K signaling under these conditions (Fig. 5 F). Whereas neither esiRNA nor EGF alone significantly reduced the effect of InlB in Lm entry (Fig. $5 \mathrm{G}$ ), the addition of both esiRNA and EGF inhibited the requirement of InlB for Lm entry into Jar cells (Fig. $5 \mathrm{H}$ ). Therefore, preactivation of PI3-K signaling in Jar cells renders InlB dispensable for $L m$ invasion.

As $L m$ EGD carries a point mutation in the gene encoding the transcriptional regulator PrfA, leading to a constitutive expression of several major virulence genes, including inl $A$ and inlB, we performed, as controls, invasion assays with EGDe, another widely used $L m$ reference strain, that express WT PrfA (Bécavin et al., 2014). We observed a similar invasion pattern: $\Delta$ inlA entry is reduced in both LS174T and Jar cells, whereas $\Delta$ inlB entry is only reduced in Jar cells (Fig. $6 \mathrm{~A}$ ). InlB-dependent PI3-K activation was also confirmed by immunoblotting (Fig. 6, B and C). In addition, the intestinal

images of LS174T and Jar cells stained with E-cadherin (red) and Fox01 (green) and Hoechst (blue). (right) Control P-Akt staining. Small left panels show separated channels and merge of boxed regions. Magnification for insets of boxed regions: 75\%. (A and E) Microscopy results were representative of at least two independent mice. (B and $D)$ Immunoblots were performed at least 2 times. Bars: (A and E) $50 \mu \mathrm{m}$. 

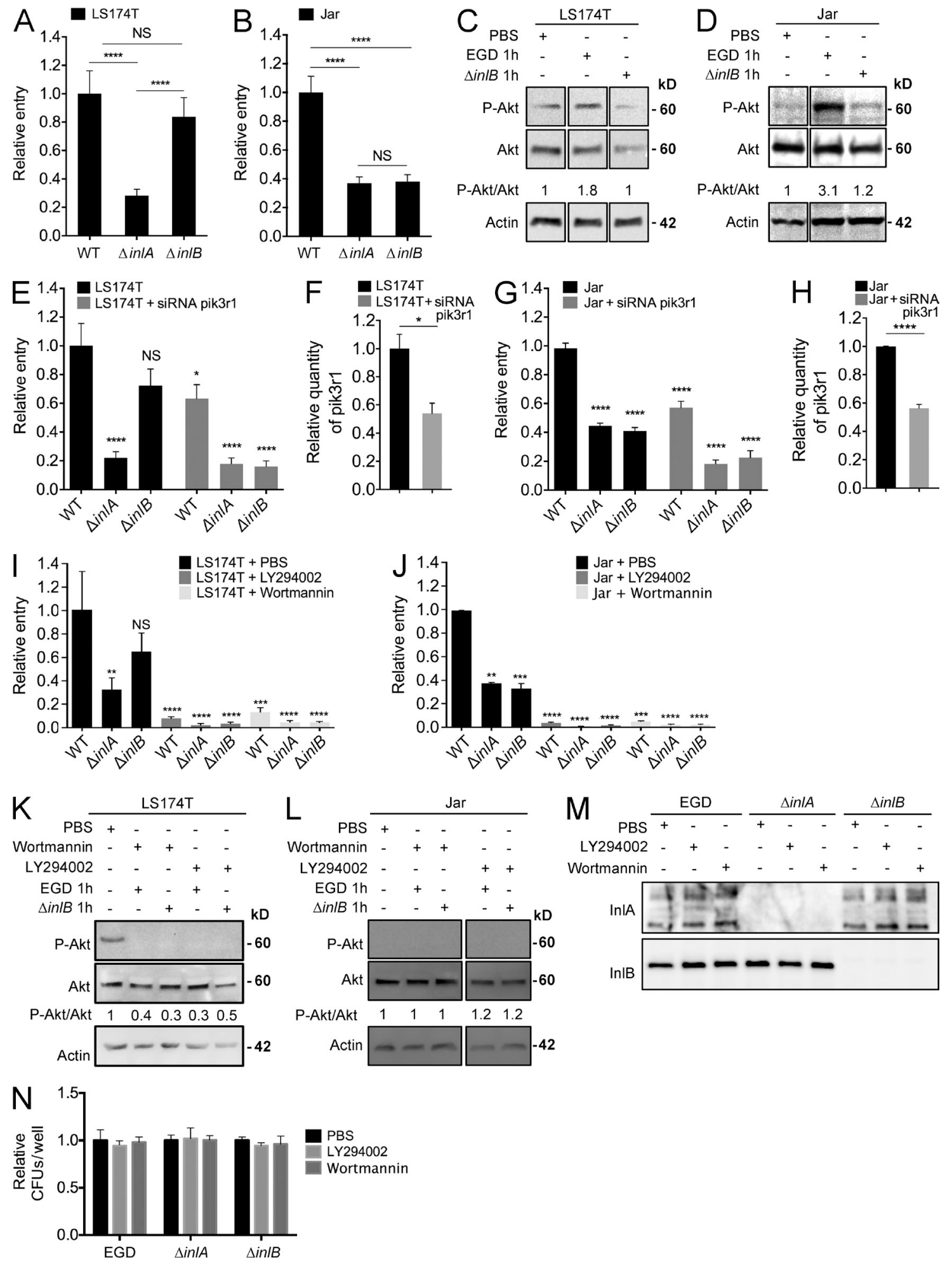

Figure 4. InIB is required for $L m$ entry in the placental Jar cell line but is dispensable for entry in the intestinal LS174T cell line, and $L m$ entry in LS174T and Jar cells is PI3-K dependent. (A) Invasion assays of WT Lm EGD and isogenic mutants $\Delta$ inlA and $\Delta$ in/B in LS174T cells. (B) Invasion assays of WT $L m E G D$ and isogenic mutants $\Delta$ inIA and $\Delta$ inIB in Jar cells. (C) Whole cell extracts from LS174T cells infected with WT $L m$ EGD and isogenic mutant $\Delta i n / B$, immunoblotted for P-Akt and total Akt. (D) Whole-cell extracts from Jar cells infected with WT $L m$ EGD and isogenic mutant $\Delta$ in/B, immunoblotted for P-Akt and total Akt. (E) Invasion assays of WT Lm EGD and isogenic mutants $\Delta$ inIA and $\Delta$ in/B in LS174T cells transfected with the Scrambled (control, black bars) or the siRNA pik3r1 (50 nM). (F) Whole cell extracts from LS174T cells transfected with the Scrambled (control, black bar) or the siRNA pik3r1 (50 nM; gray bar), where the relative quantity of pik3r1 mRNA is shown. (G) Invasion assays of WT $L m$ EGD and isogenic mutants 
invasion of $\Delta i n l A$ is significantly reduced in vivo in KIE16P mice, whereas the $\Delta$ inlB intestinal invasion was similar to the WT EGDe (Fig. 6 D).

Collectively, these results demonstrate that host cell basal PI3-K activity is critically linked to the requirement of InlB in $L m$ cell entry: InlB can promote $L m$ InlA-mediated invasion of host cells lacking intrinsic PI3-K activity, whereas it is dispensable for $L m$ entry in cells that exhibit a high basal PI3-K activity before infection.

\section{InIB is not involved in $\mathbf{L m}$ crossing of the intestinal barrier}

We have previously shown that InlB is not involved in the crossing of the intestinal barrier in hEcad transgenic mice and gerbils, which are permissive to both InlA and InlB, when assessing the bacterial load at the global intestinal tissue level (Khelef et al., 2006; Disson et al., 2008). However, Pentecost et al. (2010) have reported that InlB plays a role in invading the tip of intestinal villi in WT mice inoculated with Lm expressing a murinized version of InlA $\left(L m-\operatorname{InlA}^{\mathrm{m}}\right)$, which interacts with mEcad. Importantly, we have recently shown that $L m$-InlA ${ }^{m}$ not only invades cells expressing acc-Ecad, such as GCs, but also targets villous $\mathrm{M}$ cells, as a result of the spurious interaction of $\mathrm{Inl}^{\mathrm{m}}$ with $\mathrm{N}$-cadherin $(\mathrm{Ncad})$, which is luminally accessible at the apical pole of these cells (Tsai et al., 2013). As villous $M$ cells are especially abundant in the tips of intestinal villi (Jang et al., 2004), we reasoned that the role of InlB described by Pentecost et al. (2010) might account for the nonphysiological targeting of villous $\mathrm{M}$ cells by InlA ${ }^{\mathrm{m}}$-expressing bacteria. This led us to investigate the level of Akt phosphorylation in villous $M$ cells in vivo. As in enterocytes, and in sharp contrast to GCs, no P-Akt immunolabeling is detectable in villous $\mathrm{M}$ cells (Fig. 7, A and B), likely reflecting a low basal level of PI3-K activity in these cells and therefore supporting a role for InlB in their invasion by $L m$-InlA ${ }^{m}$. To test this hypothesis, we inoculated WT mice with either $\triangle i n l A$ or $\triangle i n l A B$ double mutant complemented with inl $A^{m}$ to investigate the role of InlB in $L m$-InlA ${ }^{\mathrm{m}}$ intestinal invasion. Strikingly, in an $\mathrm{InlA}^{\mathrm{m}}$ context, InlB promoted entry into villous $M$ cells, but was not involved in entry into GCs and other intestinal epithelial cells (Fig. 7, $\mathrm{C}$ and D). Importantly, this phenotype is confined to InlA $\mathrm{A}_{-}$ expressing $\mathrm{Lm}$, as InlA does not mediate entry into villous M cells (Tsai et al., 2013).
It has also been reported that InlB plays a role in Lm Peyer's patch invasion of WT mice, which are not permissive to InlA (Chiba et al., 2011). As $L m$ is able to invade Peyer's patches through $M$ cells (Chiba et al., 2011), we assessed $\mathrm{P}-$ Akt level in these cells. As in villous $\mathrm{M}$ cells, P-Akt expression is low in Peyer's patch $\mathrm{M}$ cells (Fig. $8 \mathrm{~A}$ ), reflecting a low basal PI3-K activity in these cells. We next performed ligated loop assays both in WT mice (which are not permissive to InlA) and in KIE16P mice (which are permissive to InlA) with either WT $L m$ or $\Delta i n l B$ strains. Similar to Chiba et al., we observed a role for InlB in invading Peyer's patches and no effect on intestinal villi in both jejunum and ileum in nonpermissive WT mice (Fig. 8 B; Chiba et al., 2011). However, in a model permissive to InlA (KIE16P mice), we observed no significant role of InlB at both intestinal villi and Peyer's patches levels (Fig. 8 C).

Altogether, these results show that InlB is not involved in the invasion of the villous intestinal epithelium in both permissive and nonpermissive mouse models but is required to invade intestinal Peyer's patches only in InlA-nonpermissive WT mice and not in InlA-permissive host such as KIE16P mice, in which InlA likely masks InlB minor contribution. These results therefore confirm that in in vivo models in which InlA-dependent entry is functional, InlB is not involved in the crossing of the intestinal barrier.

\section{InIB is involved in $L m$ crossing of the placental barrier}

To study the role of InlB in Jar cell invasion in vitro in human samples, we investigated this phenotype in vivo in human placental explants. Because maternal-fetal listeriosis most commonly occurs during the second and third trimesters (Girard et al., 2014; Kourtis et al., 2014), we used placental villous explants obtained from cesarean section deliveries at the end of uncomplicated full-term pregnancies, as described in a previous study (Lecuit et al., 2004). PI3-K expression level was quantified by confocal microscopy: P-Akt expression was low in all trophoblastic cells although it was slightly higher in cytotrophoblast cells than in syncytiotrophoblast (Fig. 9, A and B). Ecad and c-Met were also expressed in termplacenta villous trophoblast (Fig. 9, A and C).

We next investigated bacterial adhesion to and invasion of ex vivo inoculated placental explants by immunofluorescence on whole mount tissue. Intracellular WT Lm were mostly

$\Delta$ inlA and $\Delta$ inlB in Jar cells transfected with the Scrambled (control, black bars) or the siRNA pik3r1 $50 \mathrm{nM}$. (H) Whole cell extracts from Jar cells, transfected with the Scrambled (control, black bar) or the siRNA pik3r1 $50 \mathrm{nM}$ (gray bar), where the relative quantity of pik3r1 mRNA is shown. (I) Invasion assays of WT Lm EGD and isogenic mutants $\Delta$ inIA and $\Delta$ inIB in LS174T cells incubated with PBS, 100 nM wortmannin, or $50 \mu M$ LY294002. (J) Invasion assays of WT Lm EGD and isogenic mutants $\Delta$ inlA and $\Delta$ inlB in Jar cells, incubated with PBS, $100 \mathrm{nM}$ wortmannin, or $50 \mu \mathrm{M}$ LY294002. (K) Whole cell extracts from LS174T cells, incubated with PBS, $100 \mathrm{nM}$ wortmannin, or $50 \mu \mathrm{M}$ LY294002, infected with WT Lm EGD and isogenic mutant $\Delta$ in/B, and immunoblotted for P-Akt and total Akt. (L) Whole cell extracts from Jar cells, incubated with PBS, 100 nM wortmannin, or $50 \mu M$ LY294002, infected with WT $L m$ EGD and isogenic mutant $\Delta$ in $/ B$, and immunoblotted for P-Akt and total Akt. (M) Immunoblot for InIA and InIB of WT $L m$ EGD and isogenic mutants $\Delta$ inIA and $\Delta$ inIB incubated with PBS, wortmannin $100 \mathrm{nM}$ or LY294002 $50 \mu \mathrm{M}$. Associated relative CFUs per well are shown in N. (A, B, E, G, I, and J) Invasion assays were performed in triplicates and represent at least three independent experiments for each condition tested. Relative entry represents the ratio of CFUs for each strain divided by the mean of CFUs for the WT Lm EGD strain. A one-way ANOVA test followed by a Bonferroni's multiple comparisons was performed. Error bars, SEM. (C, D, K, L, and M) Immunoblots were performed at least two times. Densitometry was performed and the ratio of P-Akt over total Akt is relative to the PBS, for which the value is normalized to 1. 

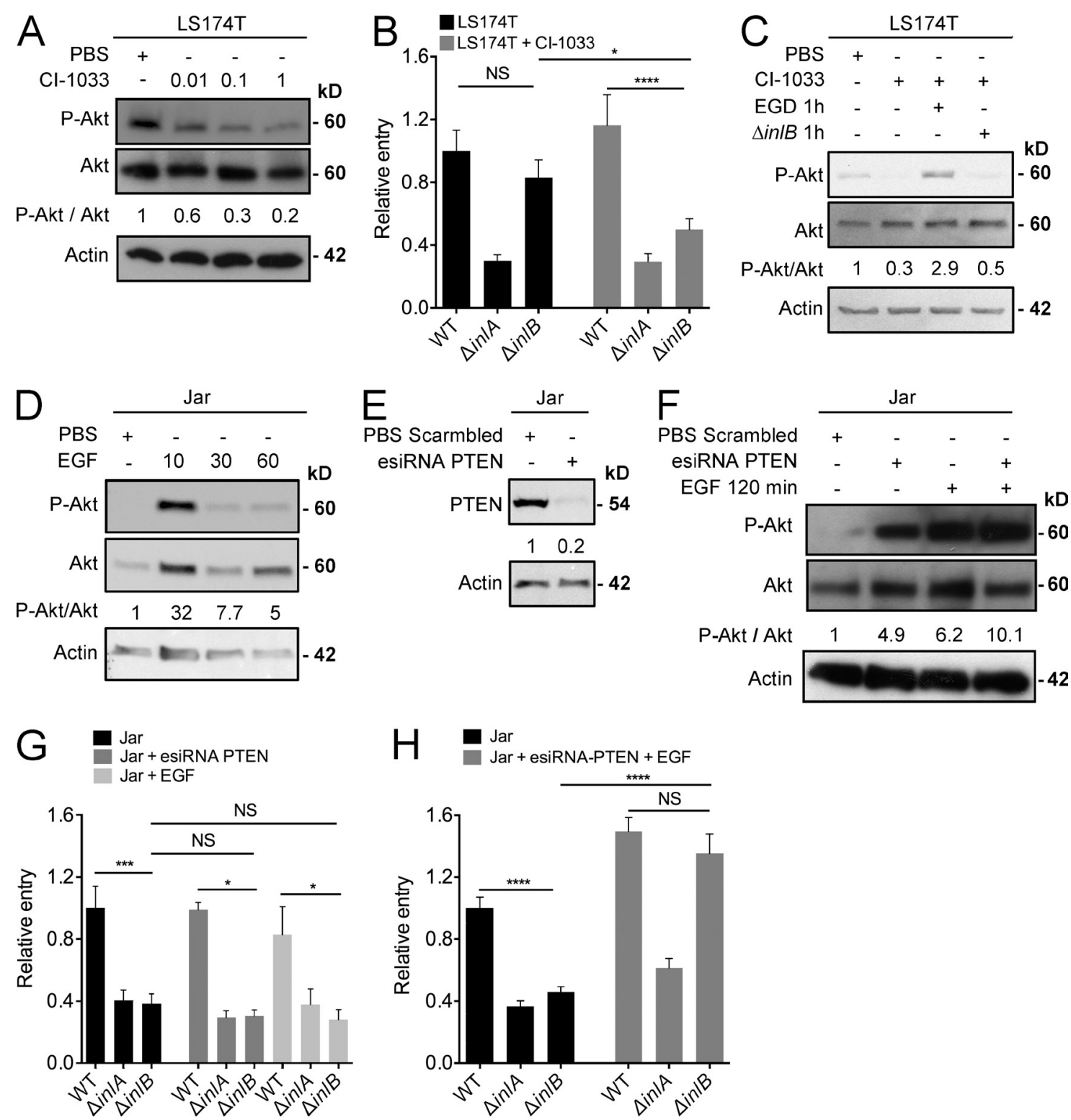

Figure 5. Modulation of PI3-K activity inversely impacts InIB-requirement for $\mathbf{L m}$ entry in vitro. (A) Whole cell extracts from LS174T cells incubated with PBS or different concentrations of the ErbB inhibitor Cl-1033 (0.01, 0.1, or $1 \mu \mathrm{M})$ during 24 h, immunoblotted for P-Akt and total Akt. (B) Invasion assays of WT Lm EGD and isogenic mutants $\Delta$ inIA and $\Delta i n / B$ in LS174T cells incubated with PBS (controls, black bars) or Cl-1033 $1 \mu \mathrm{M}$ (gray bars) for $24 \mathrm{~h}$. (C) Whole cell extracts from LS174T cells incubated with PBS or Cl-1033 $1 \mu \mathrm{M}$ and infected with WT Lm EGD and isogenic mutant $\Delta$ in/B, immunoblotted for P-Akt and total Akt. (D) Whole cell extracts from Jar cells incubated with PBS or EGF $50 \mathrm{ng} / \mathrm{ml}$ during 10, 30, or 60 min, immunoblotted for P-Akt and total Akt. (E) Whole cell extracts from Jar cells transfected with esiRNA PTEN, immunoblotted for PTEN. (F) Whole cell extracts from Jar cells transfected with esiRNA PTEN or control scrambled and incubated with PBS or EGF $50 \mathrm{ng} / \mathrm{ml}$ for $120 \mathrm{~min}$, immunoblotted for P-Akt and total Akt. (G) Invasion assays of WT $L m E G D$ and isogenic mutants $\triangle$ inlA and $\triangle$ in/B in Jar cells incubated with PBS, or transfected with esiRNA PTEN or incubated with EGF $50 \mathrm{ng} / \mathrm{ml}$. (H) Invasion assays of WT Lm EGD and isogenic mutants $\Delta i n / A$ and $\Delta$ in/B in Jar cells incubated with PBS or incubated with EGF $50 \mathrm{ng} / \mathrm{ml}$, and transfected with esiRNA PTEN. ( $B, G$ and $H)$ Invasion assays were performed in triplicates and represent at least three independent experiments for each condition tested. Relative entry represents the ratio of CFUs for each strain divided by the mean of CFUs for the WT Lm EGD strain. A one-way ANOVA test followed by a Bonferroni's multiple comparisons was performed. Error bars, SEM. (A, C, D, E, and F) Immunoblots were performed at least two times. Densitometry was performed and the ratio of P-Akt over total Akt is relative to the PBS, for which the value is normalized to 1.

detected in the syncytiotrophoblast and, to a lesser extent, in the cytotrophoblast, and $\triangle i n l A$ and $\triangle$ inlB mutant invasiveness in the syncytiotrophoblast and the cytotrophoblast was severely impaired (Fig. 10, A and B). In addition, as compared with WT Lm, the $\triangle$ inlA mutant attachment to the trophoblastic surface was reduced, whereas the $\Delta$ inlB mutant attachment to the trophoblastic surface was similar to WT Lm (Fig. 10, A and B). Finally, we performed gentamicin survival assays 

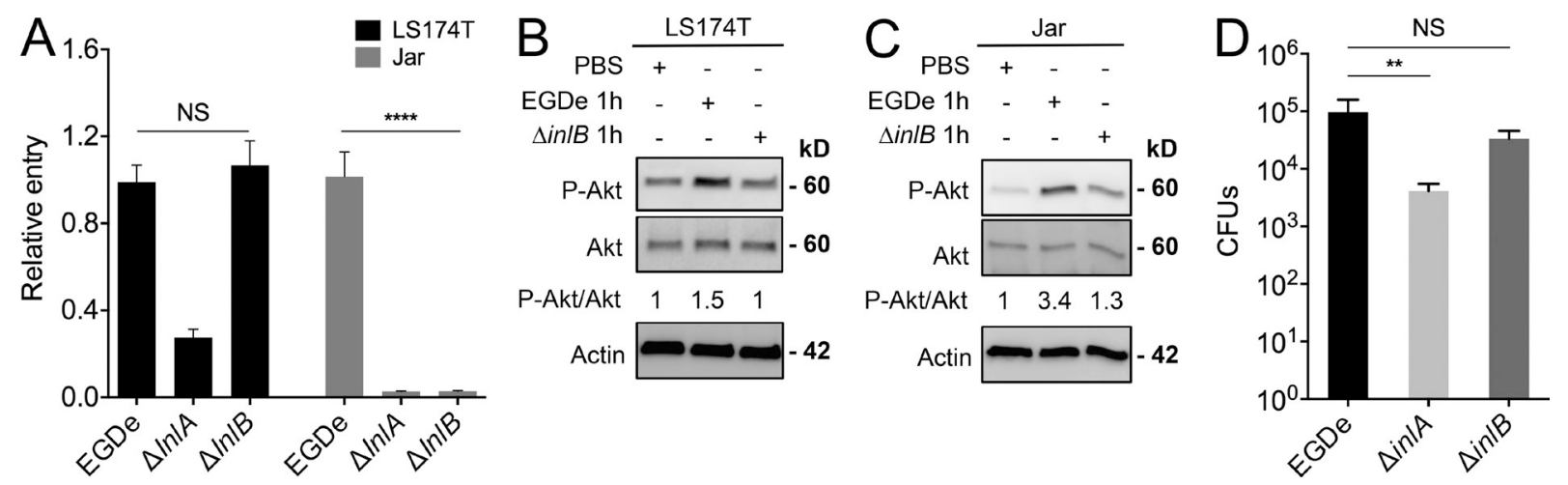

Figure 6. Wt $L m$ EGDe and isogenic mutants $\triangle i n I A$ and $\triangle i n / B$ invasion pattern is similar to WT $L m$ EGD and isogenic mutants, in vitro in LS174T and Jar cells and in vivo in KIE16P mice. (A) Invasion assays of WT Lm EGDe and isogenic mutants $\Delta$ inlA and $\Delta$ in/B in LS174T (black bars) and Jar cells (gray bars). Invasion assays were performed in triplicates and represent at least three independent experiments for each condition tested. Relative entry represents the ratio of CFUs for each strain divided by the mean of CFUs for the WT Lm EGDe strain. A one-way ANOVA test followed by a Bonferroni's multiple comparisons was performed. Error bars, SEM. (B) Whole cell extracts from LS174T cells incubated with PBS or infected with WT Lm EGDe and isogenic mutant $\Delta$ in $/ B$ and immunoblotted for P-Akt and total Akt. (C) Whole cell extracts from Jar cells incubated with PBS, or infected with WT $L m$ EGDe and isogenic mutant $\Delta$ in/B, immunoblotted for P-Akt and total Akt. Immunoblots were performed at least two times. Densitometry was performed and the ratio of P-Akt over total Akt is relative to the PBS, for which the value is normalized to 1. (D) In vivo intestinal invasion assays of WT $L m$ EGDe and isogenic mutants $\Delta$ inIA and $\Delta$ inIB. Mice were sacrificed $24 \mathrm{~h}$ after inoculation ( $n=6$ mice for each condition). A one-way ANOVA test followed by a Kruskal-Wallis multiple comparisons was performed. Errors bars, SEM.

and found, as we previously reported (Lecuit et al., 2004), a significant role for InlA and InlB in third term placental villous explant invasion (Fig. $10 \mathrm{C}$ ). Invasion of WT, $\Delta i n l A$, and $\Delta$ inlB strains were PI3K dependent because it was completely abolished when invasion assays were performed with the PI3-K irreversible inhibitors LY294002 and wortmannin (Fig. 10, D and E).

\section{DISCUSSION}

The crossings of the intestinal and placental barriers by $\mathrm{Lm}$ are the critical events that lead to bacterial access to the maternal systemic circulation and the fetal-placental unit, respectively. Studying the mechanisms by which $L m$ crosses these barriers is therefore crucial to understanding the pathophysiology of listeriosis. We have previously shown that InlA-Ecad interaction is necessary and sufficient for $L m$ crossing of the intestinal barrier and uncovered the essential and interdependent roles of InlA-Ecad and InlB-c-Met interactions in $\mathrm{Lm}$ crossing of the placental barrier (Lecuit et al., 2001; Khelef et al., 2006; Disson et al., 2008; Nikitas et al., 2011). So far, the molecular mechanisms underlying these differences have not been investigated and are not understood. It has been shown that InlA-dependent entry requires PI3-K activity and InlBc-Met interaction activates PI3-K (Ireton et al., 1996). We therefore hypothesized that InlB might be involved in InlAdependent entry via its action on PI3-K signaling.

Our in vivo observations in mice support this hypothesis: InlB is dispensable for $L m$ crossing of the intestinal barrier (Khelef et al., 2006; Disson et al., 2008) and intestinal cells targeted by $L m$, i.e., GCs, ECs, and EEFs, coexpress acc-Ecad (Lecuit et al., 2001; Khelef et al., 2006; Disson et al., 2008; Nikitas et al., 2011) and a high level of P-Akt, consistent with a constitutive PI3-K activity. In contrast, InlB is required for the crossing of the placental barrier (Lecuit et al., 2001; Khelef et al., 2006; Disson et al., 2008; Nikitas et al., 2011), where almost no P-Akt is detected. The same holds true in human placenta, where InlB is necessary to invade the trophoblast in ex vivo term placental villous explants. To address the functional relationship between InlB requirement and PI3-kinase activity, we used in vitro human intestinal (LS174T) and placental cell lines (Jar), which exhibit a level of PI3-K activity similar to intestinal and placental target cells, respectively. These in vitro models allowed us to demonstrate that (i) the experimental down-regulation of PI3-K signaling before infection reveals a role for InlB in intestinal cell invasion, and that (ii) the induction of PI3-K signaling makes InlB dispensable to invade placental cells. Together, these results reveal that InlB renders host placental cells permissive to InlA-mediated invasion in a PI3-K-dependent manner. They also provide a molecular explanation for the respective contributions of InlA and InlB on intestinal and placental barriers crossing, depending on the steady-state PI3-K activity in host barriers cells targeted by $L m$ in vivo.

We have also identified a direct in vivo correlation between Ecad accessibility on intestinal epithelial $\mathrm{Lm}$ target cells and their intrinsic PI3-K activity. Perez et al. (2008) proposed that in stable and cohesive epithelia, PI3-K activity is downregulated to allow the maintenance of Ecad interactions at cell-cell junctions, whereas in more dynamic epithelia, actin cytoskeleton remodeling requires PI3-K activity to disrupt adherens junctions and allow the formation of new sites of Ecad homophilic interactions. Our in vivo findings fit with this model: luminal accessibility of Ecad correlates with high PI3-K activity in intestinal GCs, ECs, and EEFs, where adherens junctions are dynamic (Madara et al., 1980; Madara and Trier, 1982; Kölsch et al., 2007; Marchiando et al., 2011). Indeed, 


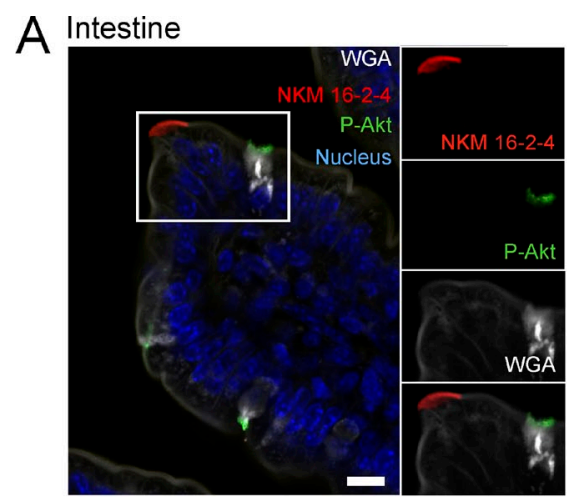

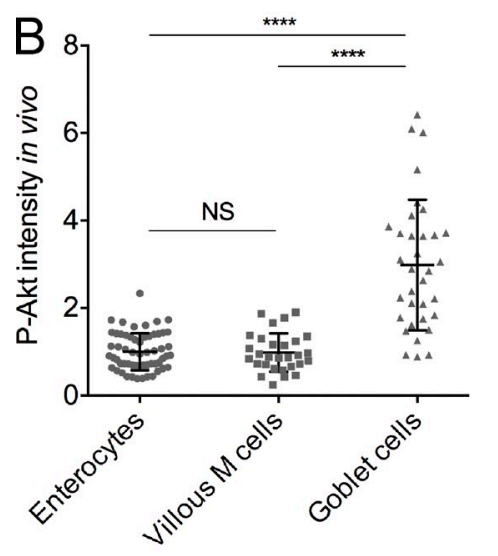
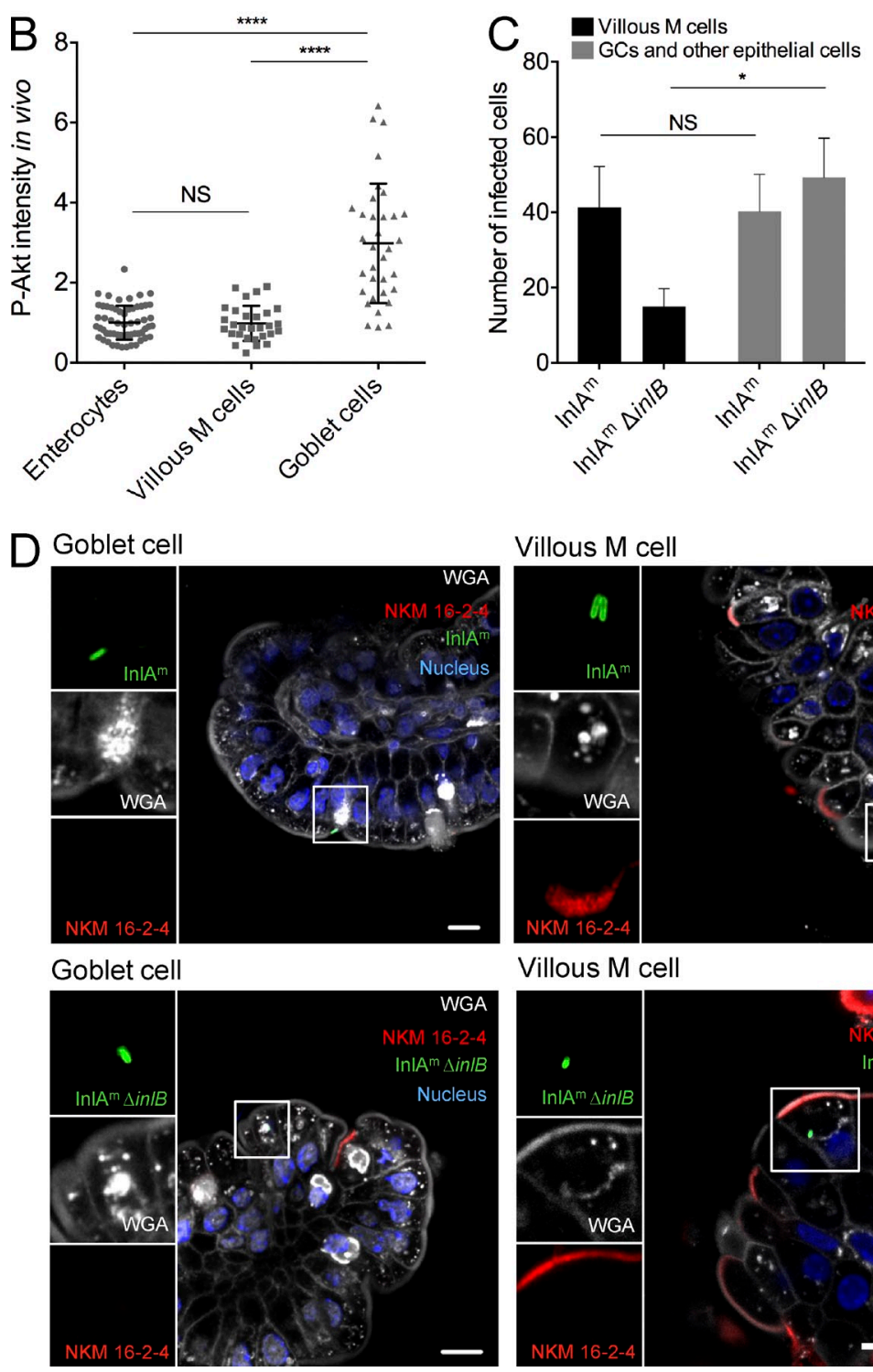

\begin{abstract}
Villous $\mathrm{M}$ cell
\end{abstract}

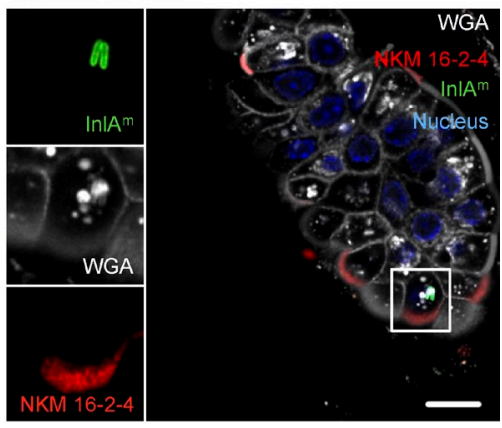

Villous M cell

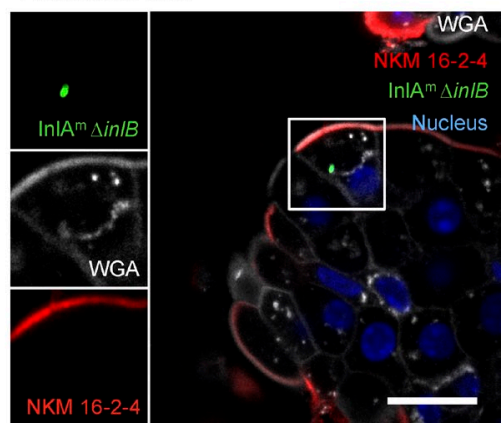

Figure 7. InIB is required for $L m$ entry in villous $M$ cells, which exhibit a low level of Akt phosphorylation. (A) Representative confocal microscopy images of a vibratome section of intestinal villous tips taken from uninfected C57BL/6 mice stained for M cells (red), P-Akt (green), WGA (white) and Hoechst (blue). Small right panels show separated channels and merge of boxed region. (B) Quantification of $\mathrm{P}$-Akt intensity in vivo, in enterocytes, villous M cells and GCs measured from at least 15 fields ( $n=3$ independent mice). Each dot corresponds to one quantified cell and at least 30 cells were quantified per cell subset. A one-way ANOVA test followed by a Bonferroni's multiple comparisons was performed. Error bars, SD. Values of P-Akt intensity are expressed as the fold-change of intensity relative to enterocytes, for which the value was normalized to 1 . (C) $\mathrm{C} 57 \mathrm{BL} / 6$ mice were orally inoculated by $10^{9} \mathrm{Lm}$-in $/ \mathrm{A}^{\mathrm{m}}$ $\Delta \operatorname{in} \mid A\left(\right.$ in $\left.\mid A^{m}\right)$ and $10^{9} L m$-inl $\mid A^{m}-\Delta$ in $\mid A B\left(\right.$ in $\mid A^{m} \Delta$ in $\left.\mid B\right)$, and sacrificed $5 \mathrm{~h}$ after inoculation. Bacterial strains location in villous $M$ cells, GCs, and other epithelial cells is shown. At least 25 villi from 3 mice were counted in each set. A one-way ANOVA test followed by a Bonferroni's multiple comparisons was performed. Error bars, SEM. (D) Representative confocal microscopy images of whole-mount intestinal villi sections taken from C57BL/6 mice stained for M cells (red), WGA (white), Lm (green), and Hoechst (blue). (left) Separated channels of boxed regions. Magnification for insets of boxed regions: 100\%. (A and D) Microscopy results were representative of at least 3 independent mice. Bars, $10 \mu \mathrm{m}$. similar to ECs and EEFs, mucus expelling-GCs undergo extensive shape changes accompanied by cell-cell junction reorganization (Hull and Staehelin, 1976; Porvaznik et al., 1983; Nikitas et al., 2011). Moreover, epithelial cell differentiation in GCs also involves PI3-K signaling cascade (Durual et al.,
2005; Wang et al., 2008). These observations suggest that, at the intestinal level, $L m$ takes advantage of adherens junction reorganization to target acc-Ecad in an InlA-dependent manner and to be internalized without the need for additional activation of PI3-K signaling, thus making it independent of InlB. 


\section{A Peyer's patch}
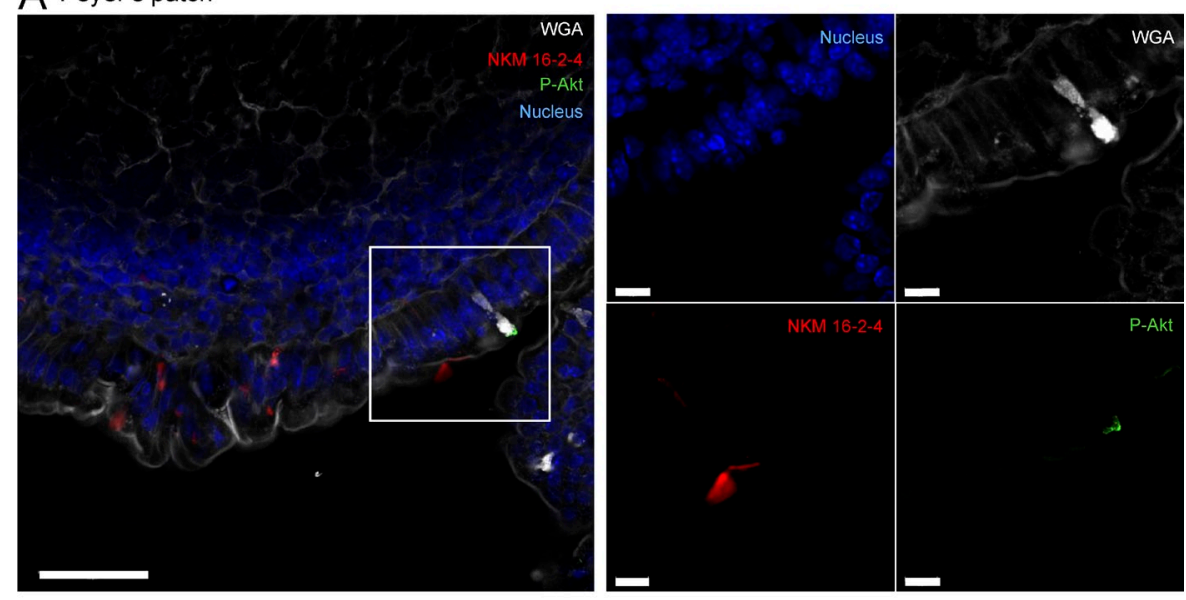

B

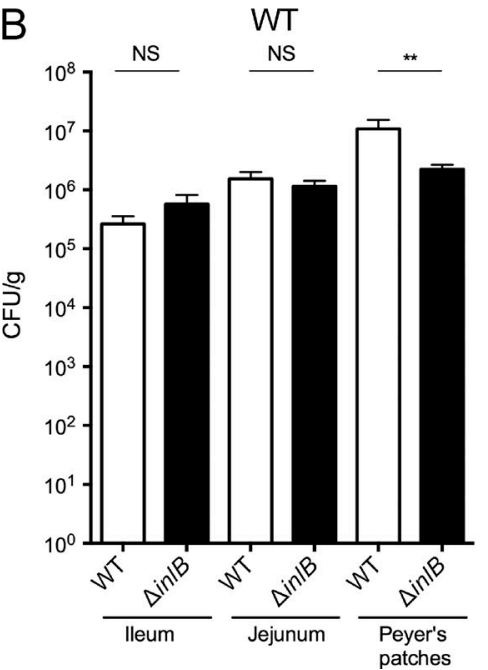

C

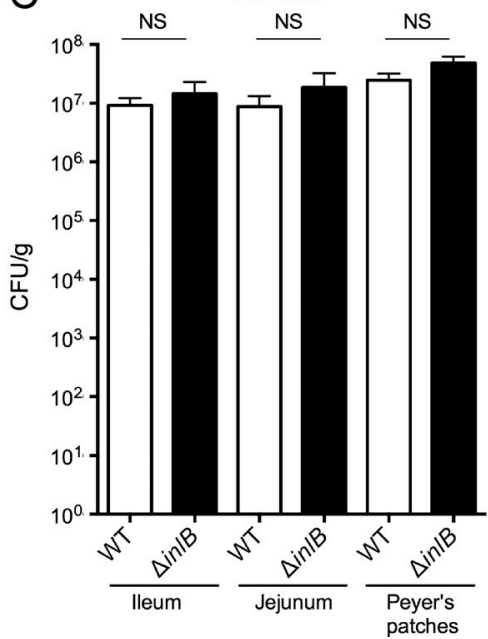

Figure 8. $\quad$ InIB is required for the crossing of Peyer's patch in WT mice nonpermissive to orally acquired listeriosis but does not play a role in permissive KIE16P mice. (A) Representative confocal microscopy images of a vibratome section of intestinal Peyer's patch, take from uninfected KIE16P mice, stained for M cells (red), P-Akt (green), WGA (white), and Hoechst (blue). (right) Separated channels of boxed region. Magnification for insets of boxed region: 75\%. Microscopy results were representative of at least three independent mice. Bars: (left) $50 \mu \mathrm{m}$; (right) $10 \mu \mathrm{m}$. (B) Ligated loop assays in BALB/c mice infected with WT $L m$ (EGD) and $L m-\Delta$ in $/ B$. $\mathrm{CFU} / \mathrm{g}$ in jejunum, ileum, and Peyer's patches are shown. (C) Ligated loop assays in KIE16P mice infected with WT $L m$ (EGD) and $L m-\Delta$ inlB. CFU/g in jejunum, ileum, and Peyer's patches are shown. $n=9$ mice for each condition. A Mann-Whitney $U$ test was performed. Error bars, SEM.
There are apparently conflicting data regarding the role of InlB in the crossing of intestinal barrier: whereas we have repeatedly found no role for InlB in $L m$ crossing of the intestinal barrier in in vivo models permissive to both InlA-Ecad and InlB-c-Met interactions (Khelef et al., 2006; Disson et al., 2008), it has been reported by others that InlB accelerates invasion at villous tips in an experimental model using murinized InlA (Pentecost et al., 2010) and plays a role at the Peyer's patch level in WT mice (Chiba et al., 2011). Here, we have confirmed these results and revisited their interpretations in light of our new investigations: we compared the infection of murinized InlA (InlA ${ }^{\mathrm{m}}$ ) expressing $\mathrm{Lm}$ (Wollert et al., 2007) in WT mice (Pentecost et al., 2010) and the infection of WT Lm in KIE16P mice expressing humanized mEcad (Disson et al., 2008) at the villous tip level. Importantly, we have recently demonstrated that InlA ${ }^{m}$ confers to $L m$ the ability to target both Ecad and Ncad, the latter being accessible on the luminal side of villous M cells (Tsai et al., 2013). As a consequence, InlA ${ }^{\mathrm{m}}$-expressing $\mathrm{Lm}$ targets not only GCs, but also villous $M$ cells, a spurious portal of entry that is not observed upon InlA-mediated invasion of the intestine (Tsai et al., 2013). No P-Akt is detectable in villous $\mathrm{M}$ cells, which are particularly abundant at the tip of intestinal villi, and we have shown a critical role for InlB in villous $M$ cells invasion. These results therefore indicate that the InlB-dependent villous tip invasion observed by Pentecost et al. (2010) is likely a direct consequence of the nonphysiological targeting of villous $M$ cells by InlA ${ }^{m}$. This observation further challenges the relevance of using InlA ${ }^{\mathrm{m}}$-expressing $L m$ to study host intestinal barrier crossing. In WT mice at the Peyer's patch level, we also confirmed that InlB is involved in intestinal invasion. Chiba et al. (2011) have reported that Lm invasion occurs through Peyer's patches via M cells, which are specialized epithelial cells continuously sampling the intestinal lumen content (Neutra et al., 1996). As Peyer's patch M cells exhibit a low Akt phosphorylation staining, this InlB-dependent phenotype likely accounts for the ability of InlB-c-Met interaction to trigger PI3-K signaling and induce InlA-independent internalization (Ireton et al., 1996). In contrast, in KIE16P mice, we did not observe any role for InlB at Peyer's patch level. This suggests that, in models permissive to both InlA and InlB, InlB-requirement for Lm invasion of Peyer's patch 


\section{A Human term placental villous explant}

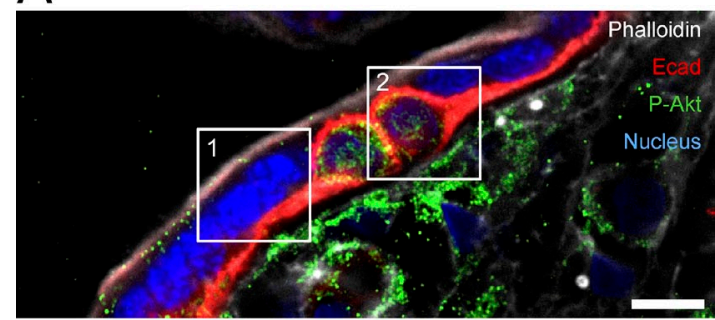

Syncytiotrophoblast
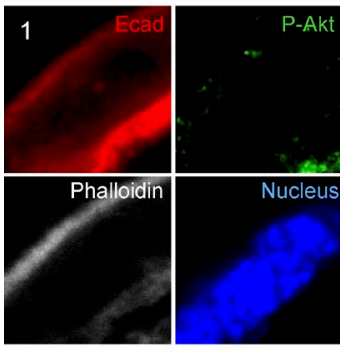

Cytotrophoblast
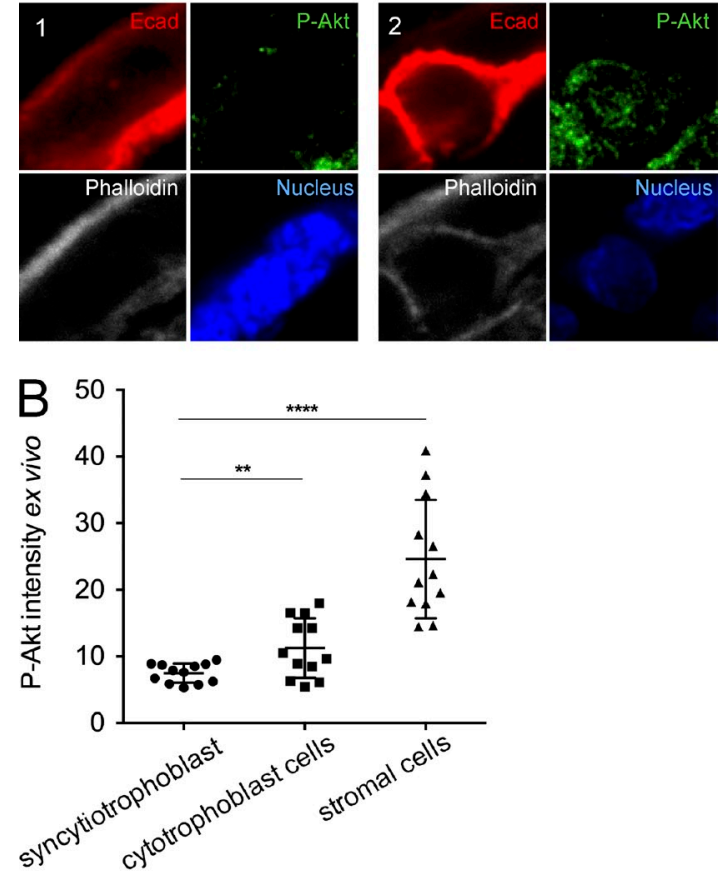

C Human term placental villous explant

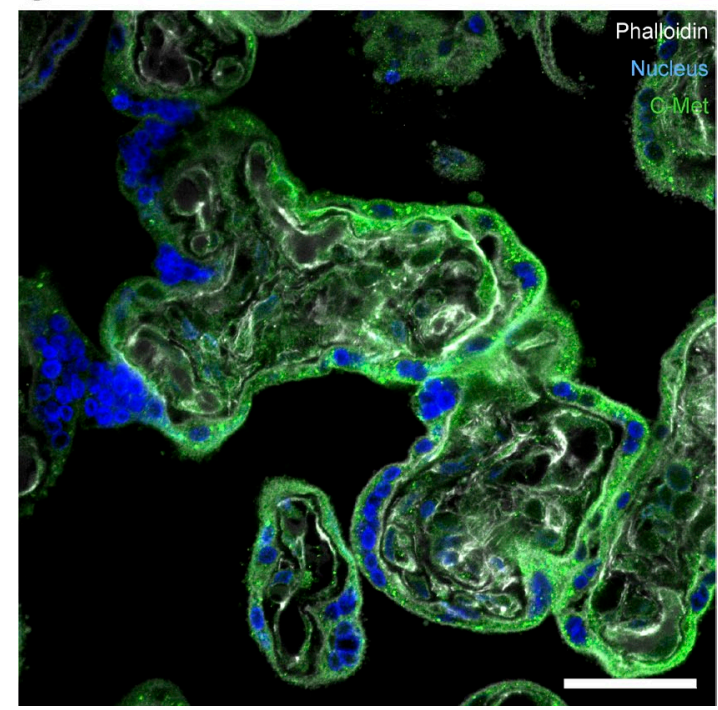

Figure 9. Syncytiotrophoblast cells from human term placental villous explants express the InIB receptor c-Met and do not exhibit phosphorylated Akt. (A) Representative confocal microscopy images of a vibratome section taken from uninfected human term placenta, stained for Ecad (red), P-Akt (green), Phalloidin (white), and Hoechst (blue). (bottom)
$M$ cells is masked by InlA-dependent translocation across the follicle-associated-epithelium (Lecuit et al., 2001; Khelef et al., 2006; Disson et al., 2008; Nikitas et al., 2011).

At the placental level, we have previously shown that $\mathrm{Lm}$ targets Ecad at the syncytiotrophoblast apical surface on third trimester human placental villi that are directly exposed to the maternal blood, whereas cytotrophoblast cells, which are located beneath the syncytiotrophoblast layer, are poorly targeted by $\operatorname{Lm}$ (Lecuit et al., 2004). By assessing bacterial trophoblastic localization we confirmed the preferential targeting of syncytiotrophoblast cells by WT Lm and reveal that InlA mediates $\mathrm{Lm}$ adhesion on syncytiotrophoblast whereas InlB does not, the latter being only involved in the internalization process.

PI3-K activity in human trophoblastic cells of ex vivo term placental villous explants was low, compared with the stromal cells located beneath the epithelium. In line with previous studies (Perez et al., 2008), the lack of classical adherens junctions in human syncytiotrophoblast is likely to be associated with reduced Ecad dynamics and a weak PI3-K-dependent cytoskeletal rearrangement. Our results in human placental explants demonstrate that human cytotrophoblast Akt is slightly phosphorylated, whereas it is not detectable in the syncytiotrophoblast. Such a low level of syncytiotrophoblast PI3-K activity explains the requirement of InlB to trigger PI3-K signaling and thereby support InlA-dependent invasion of syncytiotrophoblast to cross the placental barrier (Lecuit et al., 2004). Interestingly and consistent with the observed basal activation of PI3-K in cytotrophoblasts, Robbins et al. (2010) reported a role for InlA but no role for InlB in the invasion of cytotrophoblasts of first trimester placental organ cultures.

Host cell PI3-K signaling cascade is implicated in the internalization of a wide range of pathogens, including bacteria, virus, and parasites (Saeed et al., 2008; Bucior et al., 2012; Cummings et al., 2012). Here we report that GCs express a high basal level of PI3-K activity in vivo in uninfected conditions. Our results with $L m$ illustrate that endogenous elevated PI3-K activity can facilitate pathogen entry into these cells. Therefore, intestinal GCs may constitute a potential portal of entry for other enteroinvasive pathogens if they can achieve the initial contact, as GCs rather than the pathogen provides the internalization signaling needed for entry. These results also challenge the prevailing view that GCs only protect intestine from infection through mucus secretion in the intestinal lumen

Separated channels of boxed regions are shown, as are the lack of P-Akt in syncytiotrophoblasts (left) and the presence of P-Akt in cytotrophoblast (right). Magnification for insets of boxed regions: 50\%. (B) Quantification of P-Akt intensity ex vivo in human term placenta, measured from at least 12 fields ( $n=2$ independent experiments). A one-way ANOVA followed by a Bonferroni's multiple comparisons test was performed. Error bars, SD. (C) Representative confocal microscopy images of a vibratome section taken from uninfected human term placental villous explant, stained for c-Met (green), phalloidin (white), and Hoechst (blue). ( $A$ and $C$ ) Microscopy results were representative of at least three independent human placental explants. Bars: (A) $20 \mu \mathrm{m}$; (C) $50 \mu \mathrm{m}$. 
A Human term placental villous explant
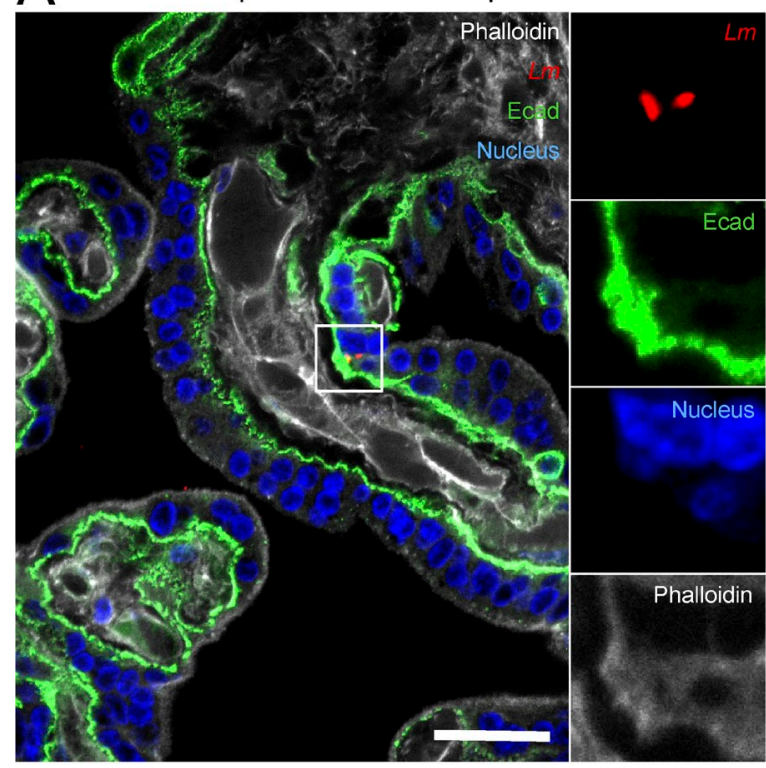

Nucleus

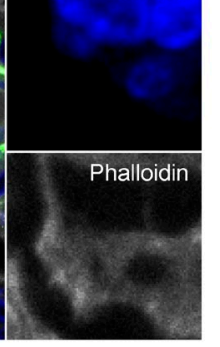

$\mathrm{B}_{25} \square \mathrm{WT} \square \operatorname{\Delta inlA} \square \operatorname{\Delta inlB}$
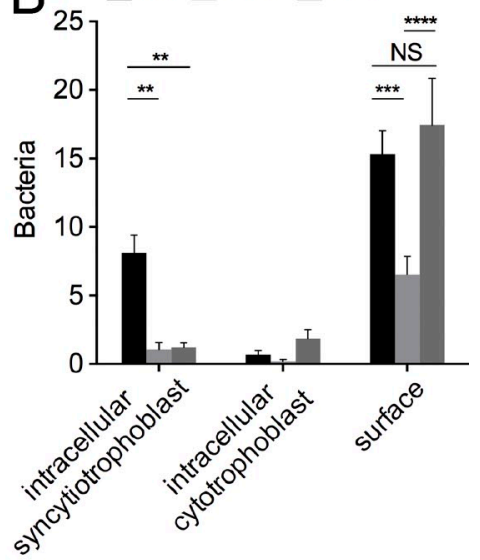
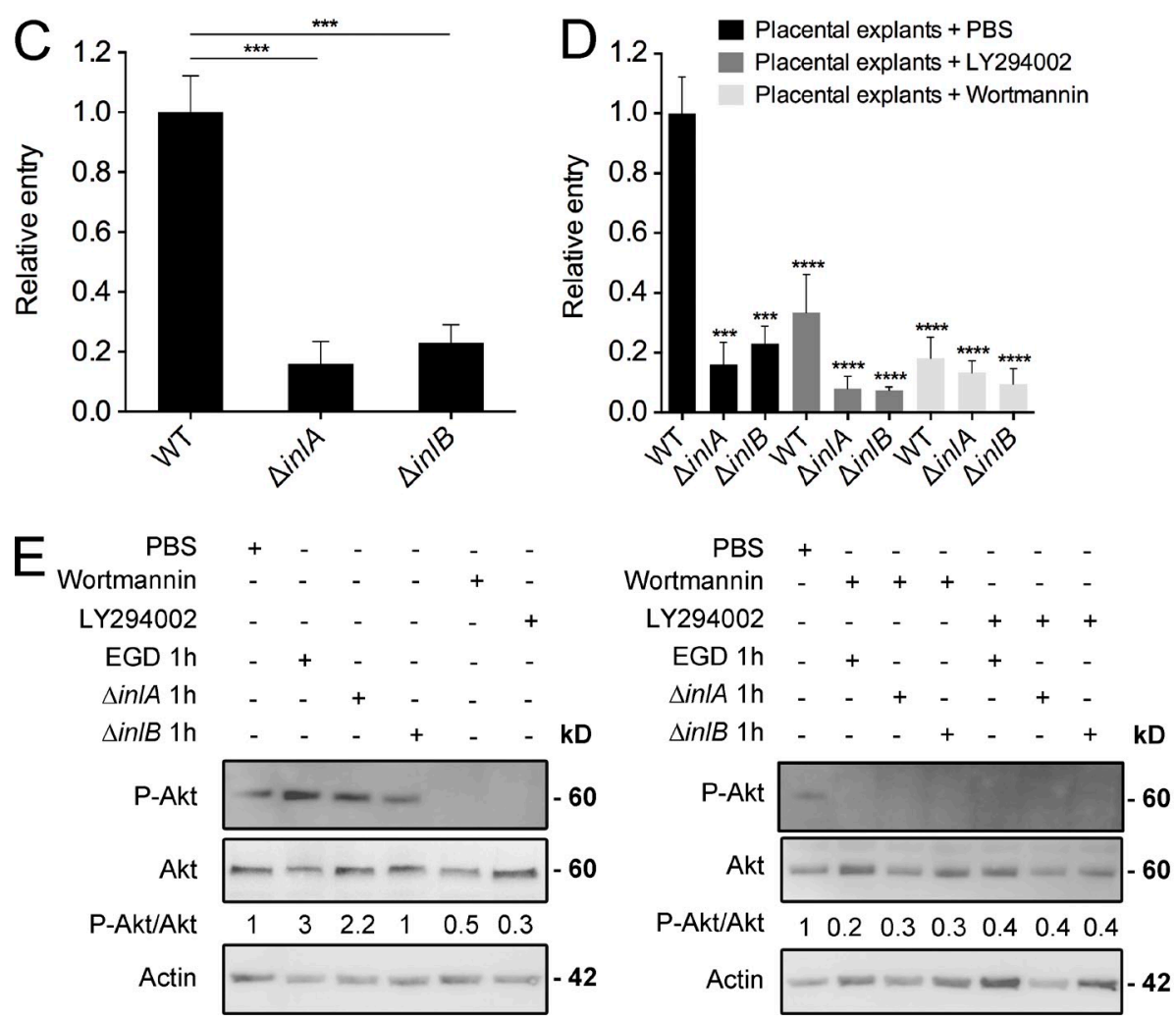

Figure 10. Lm invasion of human term placental villous explants is PI3-K and InIB dependent. (A) Representative confocal microscopy images of a vibratome section take from ex vivo inoculated human term placenta, stained for Ecad (green), WT Lm (red), phalloidin (white), and Hoechst (blue) showing WT Lm in the syncytiotrophoblast. (right) Separated channels of boxed region. Magnification, 200\%. Microscopy results were representative of at least three independent human placental explants. Bar, $20 \mu \mathrm{m}$. (B) Localization of WT Lm and isogenic mutants $\Delta$ inIA and $\Delta$ in/B, in human term placenta, showing intracellular bacteria (syncytiotrophoblast and cytotrophoblast) and bacteria attached to the epithelial surface. Quantification measured from at least 15 stacks of $35 \mu \mathrm{m}$ ( $n=2$ independent experiments). A two-way ANOVA test followed by a Bonferroni's multiple comparisons test was performed. Error bars, SEM. (C) Invasion assays of WT Lm EGD and isogenic mutants $\Delta$ inIA and $\Delta$ in/B in human term placental villous explants. A one-way ANOVA test followed by a Bonferroni's multiple comparisons test was performed. Error bars, SEM. (D) Invasion assays of WT $L m$ EGD and isogenic mutants $\Delta$ inIA and $\Delta$ inIB in human term placental villous explants incubated with PBS, Wortmannin $100 \mathrm{nM}$ or LY294002 $50 \mu \mathrm{M}$. A one-way ANOVA test followed by a Bonferroni's multiple comparisons test was performed. Error bars, SEM. Invasion assays were performed in triplicates and represent at least 3 independent 
(McGuckin et al., 2011) and suggest that the mucus-secreting cells may constitute a target for, rather than only a defense against, pathogens.

However, very few pathogens actively cross the placental barrier (Doran et al., 2013). As an example, Candida albicans may cross the intestinal barrier in an Als3-Ecad-dependent manner, leading to a systemic infection (Phan et al., 2007), but it has not been described to breach the placental barrier. This indicates that targeting of Ecad alone is not sufficient to promote placental invasion, and that C. albicans is likely missing an effector inducing PI3-K signaling at the placental barrier, as does Lm. Lack of junction dynamics and constitutive PI3-K activity at the syncytiotrophoblast level probably accounts for the efficiency of the syncytium as a barrier against pathogens (Robbins and Bakardjiev, 2012).

In conclusion, using $L m$ as a model to study host-pathogen interactions, we have provided a molecular explanation for the respective and complementary contributions of InlA and InlB in $L m$ crossing of host barriers, and revealed the critical role of PI3-K activity in the permissiveness of host barriers to microbial invasion. These results illustrate how microbial pathogens have evolved to invade mammalian tissues, taking advantage of both similarities and differences of host barriers. They also suggest that the absence of placental constitutive PI3-K activity may reinforce its barrier function toward pathogens, with the exception of those that have evolved ways to stimulate it exogenously, like $\mathrm{Lm}$.

\section{MATERIALS AND METHODS}

Ethics statement. Informed consent for the use of the human placenta was obtained according to the protocol n²014-13 approved by the "Comite de Recherche Clinique de l'Institut Pasteur" (CORC) and by the "Comité de protection des personnes Ile de France IV". All the procedures were in agreement with the guidelines of the European Commission for the handling of laboratory animals, directive 86/609/EEC and were approved by the Animal Care and Use Committee of the Institut Pasteur, as well as by the ethical committee of "Paris Centre et Sud" under the number 2010-0020.

Bacterial strains and culture conditions. Bacteria used in this study are Lm WT strain EGD (BUG 600), Lm EGD strain isogenic inlA deletion mutant $\Delta$ inlA (BUG 947), Lm EGD strain isogenic inlB deletion mutant $\Delta i n l B$ (BUG 1047), Lm EGD strain isogenic inlAB deletion mutant $\triangle$ inlAB (BUG 949), Lm WT EGDe (BUG 1600), Lm EGDe strain isogenic inlA deletion mutant $\triangle i n l A$ (BUG 1454), and Lm EGDe strain isogenic inlB deletion mutant $\Delta i n l B$ (BUG 1455). For the construction of $\mathrm{Lm}$ isogenic EGD strains expressing murinized InlA (InlA ${ }^{\mathrm{m}}$ ), pAD-inl $A^{m}$ (Tsai et al., 2013) was introduced to $\triangle i n l A$ and $\triangle i n l A B$ strains by conjugative mating with $E$. coli S17-1

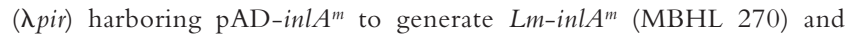
Lm-inl $A^{m} \Delta i n l B(\mathrm{MBHL} 271)$, respectively. Bacteria were cultured as previously described (Disson et al., 2009).

Cell lines and culture conditions. Jar cells (American Type Culture Collection [ATCC]; HTB 144) were used between passages 10 and 20 and grown in Roswell Park Memorial Institute Medium 1640 containing Glutamax (RPMI 1640 + Gibco), supplemented with 10 nM Hepes buffer (Gibco), $1 \mathrm{mM} \mathrm{Na}^{+}$-Pyruvate (Gibco), and 10\% fetal calf serum (Gibco). LS174T cells (ATCC CL-188) were used between passages 10 and 30 and grown in Minimum Essential Medium (MEM; Gibco) with $0.1 \mathrm{mM}$ nonessential amino acids (Gibco) and 10\% fetal calf serum (Gibco). Cells were grown at $37^{\circ} \mathrm{C}$ with $5 \% \mathrm{CO}_{2}$. Placental villous explants were used in Dulbecco's Modified Eagle Medium containing Glutamax (DMEM + Glutamax; Gibco) at $37^{\circ} \mathrm{C}$ with $5 \% \mathrm{CO}_{2}$.

Animals. We used 8-10-wk-old female C57BL/6 mice (Janvier), BALB/c mice (Charles River), and knock-in mice expressing humanized mEcad (KIE16P mice; >F10; Disson et al., 2008). Placentas were taken from 21-d pregnant mice.

Antibodies. The following antibodies were used for immunostaining and Western blotting: anti-mEcad clone ECCD-2 rat monoclonal antibody (Invitrogen), anti-hEcad clone HECD-1 mouse monoclonal antibody (Invitrogen), anti-P-Akt (Ser473) rabbit polyclonal antibody (Osenses), anti-mouse $M$ cell clone NKM 16-2-4 rat monoclonal antibody (Miltenyi Biotec), R11 anti- $L m$ rabbit polyclonal antibody (Dramsi et al., 1998), anti-c-Met SP260 rabbit polyclonal antibody (Santa Cruz laboratories), anti-FoxO1 rabbit polyclonal antibody (Cell Signaling Technology), anti-cytokeratin 18 mouse monoclonal antibody (Abcam), anti-Akt mouse monoclonal antibody (Cell Signaling Technology), anti- $\beta$-actin clone AC-15 mouse monoclonal antibody (Sigma-Aldrich), phalloidin conjugated with Alexa Fluor 647 (Invitrogen), wheat germ agglutinin (WGA) conjugated with Alexa Fluor 647 (Jackson ImmunoResearch Laboratories), Alexa Fluor 488 and 568 goat anti-rabbit (Invitrogen), Alexa Fluor 546 and 647 goat anti-rat (Invitrogen), Alexa Fluor 647 goat anti-mouse (Invitrogen), and Hoechst 33342 (Invitrogen).

Human term placental villous explants. Placentas were obtained immediately after a repeat cesarean section delivery at the end of an uncomplicated full-term pregnancy. None of the patients received antibiotics before the placental harvest. A $0.5-\mathrm{cm}$ thick section of decidual basalis was removed from the placenta, and villous tissue was dissected from multiple cotyledons, avoiding the chorionic plate. A single villous explant was then placed in each well of a 24-well plate containing DMEM and subjected to invasion assays, tissue labeling for immunofluorescence, or immunoblot.

Cell invasion assay. Gentamicin survival assays was performed as previously described (Lecuit et al., 1997). A 0.25-ml aliquot of an overnight bacterial culture was inoculated into $5 \mathrm{ml}$ of BHI liquid medium (Difco) and incubated at $37^{\circ} \mathrm{C}$ with shaking until an $\mathrm{OD}_{600}$ of 0.8 was reached. Bacteria were then recovered by centrifugation, suspended in $1 \mathrm{ml}$ of RPMI or MEM, and diluted 100-fold in culture medium without fetal calf serum. Medium from 24-well plates containing cultured cells was removed and cells were rinsed in culture medium without fetal calf serum. $1 \mathrm{ml}$ of the diluted bacterial suspension was then added per well. After 1 -h incubation at $37^{\circ} \mathrm{C}$ in $5 \% \mathrm{CO}_{2}$, cells were rinsed in culture medium with fetal calf serum and incubated for an additional $1 \mathrm{~h}$ in culture medium containing $10 \mu \mathrm{g} / \mathrm{ml}$ gentamicin (Gibco) to kill extracellular bacteria. Gentamicin-containing medium was removed, and cells were rinsed in RPMI or MEM and lysed with $0.1 \%$ Triton X-100 in PBS. The number of CFUs of intracellular bacteria was determined by plating serial dilution of the resulting lysates on BHI agar plates. The level of invasion was defined by dividing the number of viable bacteria recovered after gentamicin treatment by the number of viable bacteria that had been inoculated into each well of the 24 -well plates and normalized by the level

experiments for each condition tested. Relative entry represents the ratio of CFUs for each strain divided by the mean of CFUs for the WT $L m$ EGD strain. (E) Whole cell extracts from human term placental villous explants incubated with PBS, $100 \mathrm{nM}$ Wortmannin, or $50 \mu \mathrm{M}$ LY294002 and infected with WT Lm EGD and isogenic mutants $\triangle$ inIA and $\Delta i n / B$, and then immunoblotted for P-Akt and total Akt. Immunoblots were performed at least two times. Densitometry was performed and the ratio of P-Akt over total Akt is relative to the PBS, for which the value was normalized to 1. 
of invasion obtained with the WT Lm in the same cell type. Invasion assays were performed in triplicates and represent at least 3 independent experiments for each condition tested.

The esiRNA Human PTEN and the scrambled siRNA were purchased from Sigma-Aldrich. The esiRNAs are pools of siRNAs that all target the same mRNA sequence.

Transient transfection of Jar cells was performed with jetPRIME transfection reagent (Polyplus transfection). Cells were seeded into the 24-well plates for $24 \mathrm{~h}$, and then transfected with esiRNA Human PTEN or scrambled, followed by $24 \mathrm{~h}$ of incubation and replacement of transfection medium with growth medium another $24 \mathrm{~h}$ of incubation before infection. PBS and EGF ( $50 \mathrm{ng} / \mathrm{ml}$; Invitrogen) were added $1 \mathrm{~h}$ before $L m$ infection and during the course of the infection.

The siRNA pik3r1, which targets the type IA PI3-K p $85 \alpha$ subunit, and the scrambled siRNA were purchased from Life Technologies.

Transient transfection of LS174T and Jar cells was performed with INTERFER in and JetPRIME transfection reagent, respectively (Polyplus transfection). Cells were seeded into the 24-well plates for $24 \mathrm{~h}$, and then transfected with siRNA pik3r1 or scrambled, followed by $24 \mathrm{~h}$ of incubation and replacement of transfection medium with growth medium another $24 \mathrm{~h}$ of incubation before infection.

The pan-ErbB inhibitor CI-1033 was purchased from Biovision. LS174T cells were seeded into the 24-well plates for $24 \mathrm{~h}$, and then PBS and CI-1033 $(1 \mu \mathrm{M})$ were added for $24 \mathrm{~h}$ into the culture medium.

The wortmannin was obtained from Sigma-Aldrich and the LY294002 was purchased from Alexis Biochemicals. Jar and LS174T cells were seeded into the 24-well plates for $48 \mathrm{~h}$, and then $100 \mathrm{nM}$ wortmannin or $50 \mu \mathrm{M}$ LY294004 were added $1 \mathrm{~h}$ before $L m$ infection and during the course of the infection.

Placental villous explants invasion assay. Placental villous explants invasion assay was performed as previously described (Lecuit et al., 2004). A 0.5-ml aliquot of an overnight bacterial culture was inoculated into $10 \mathrm{ml}$ of BHI liquid medium (Difco), and incubated at $37^{\circ} \mathrm{C}$ with shaking until an $\mathrm{OD}_{600}$ of 0.8 was reached. Bacteria were then recovered by centrifugation, suspended in an equal volume of DMEM, and diluted 50-fold in DMEM, without fetal calf serum. Medium from 24-well plates containing placental villous explants was removed and tissues were rinsed in DMEM without fetal calf serum. $1 \mathrm{ml}$ of the diluted bacterial suspension was then added per well. After 1-h incubation at $37^{\circ} \mathrm{C}$ in $5 \% \mathrm{CO}_{2}$, cells were rinsed in culture medium with fetal calf serum and incubated for an additional $2 \mathrm{~h}$ in culture medium containing $10 \mu \mathrm{g} / \mathrm{ml}$ gentamicin (Invitrogen) to kill extracellular bacteria. Gentamicin-containing medium was removed and placental villous explants were rinsed three times in DMEM and disrupted by homogenization in sterile PBS. The number of CFUs of intracellular bacteria was determined by plating serial dilution of the resulting lysates on $\mathrm{BHI}$ agar plates. The level of invasion was defined by dividing the number of viable bacteria recovered after gentamicin treatment by the number of viable bacteria that had been inoculated into each well of the 24-well plates, and normalized by the level of invasion obtained with the WT Lm in the same cell type. Invasion assays were performed in triplicates and represent at least three independent experiments for each condition tested. Wortmannin (100 nM) or LY294004 $(50 \mu \mathrm{M})$ were added $1 \mathrm{~h}$ before $L m$ infection and during the course of the infection.

Cell labeling for immunofluorescence. Cells were grown on glass coverslips in 24-well plates at a density of $5 \times 10^{4}$ cells/well for $48 \mathrm{~h}$. Cells were washed with PBS supplemented with $\mathrm{Ca}^{2+}$ and $\mathrm{Mg}^{2+}$ (Invitrogen), and then fixed with 4\% paraformaldehyde (Electron Microscopy Sciences) in PBS for $1 \mathrm{~h}$ at room temperature. Cells were permeabilized for $30 \mathrm{~min}$ in PBS supplemented with $0.4 \%$ Triton X-100 (Sigma-Aldrich) and 3\% BSA (SigmaAldrich), and then labeled with the appropriate primary and secondary antibodies for $1 \mathrm{~h}$ at room temperature. Cells on coverslips were mounted in Fluoromount $\mathrm{G}$ (Interchim).

Tissue labeling for immunofluorescence. Tissues were fixed in $4 \%$ paraformaldehyde (Electron Microscopy Sciences) in PBS for $2 \mathrm{~h}$ at room temperature and either stained directly (whole mount) or embedded in $4 \%$ low-melting-point agarose (Sigma-Aldrich) for $200 \mu \mathrm{M}$ thick vibratome sections (Micro HM 650V; Thermo Fisher Scientific; Disson et al., 2009). Sections were stained in a similar manner to whole mount tissue but mounted in Fluoromount G (Interchim). Tissues were permeabilized for $1 \mathrm{~h}$ in PBS supplemented with 0.4\% Triton X-100 (Sigma-Aldrich) and 3\% BSA (SigmaAldrich) and preincubated for $1 \mathrm{~h}$ in the blocking buffer (PBS supplemented with $3 \% \mathrm{BSA}$ ). Then tissues were labeled with the appropriate primary and secondary antibodies for $2 \mathrm{~h}$ at room temperature.

For immunofluorescence of cryosections, retrieved tissues were fixed and embedded in OCT (Tissue-Tek) at $-80^{\circ} \mathrm{C}$, cut using a cryostat at $-20^{\circ} \mathrm{C}$ $(10-\mu \mathrm{m}$-thick sections), post-fixed in pure ethanol, and labeled with the appropriate primary and secondary antibody for $2 \mathrm{~h}$ at room temperature. Sections were mounted in Fluoromount $\mathrm{G}$ (Interchim).

For immunofluorescence of placental villous explants, tissues were stained in a similar manner as whole-mount tissue but were mounted in Fluoromount $\mathrm{G}$ (Interchim) and dried at $37^{\circ} \mathrm{C}$ for $24 \mathrm{~h}$.

To label accessible Ecad of uninfected tissues, whole mount tissues were directly labeled before the permeabilization step. To detect accessible mEcad we used ECCD-2, a specific rat monoclonal antibody raised against the extracellular domain of mEcad (Invitrogen).

The Ser-473 P-Akt blocking peptide was purchased from Cell Signaling Technology. Vibratome sections were preincubated for $30 \mathrm{~min}$ with the blocking peptide in PBS supplemented with $0.4 \%$ Triton X-100 and 3\% BSA, before tissue labeling.

Immunoblot. Cells were grown in a 12-well plate at a density of $2 \times 10^{5}$ cells/well for $48 \mathrm{~h}$. Cells were washed with PBS and lysed in lysis-buffer containing RIPA 1X (Sigma-Aldrich), anti-phosphatase and anti-protease (Roche). Protein concentration from total protein extracts was quantified using the BCA Protein Assay kit (Thermo Fisher Scientific). $20 \mu \mathrm{g}$ of protein for each sample was boiled for $5 \mathrm{~min}$ in NuPage buffer (Invitrogen) with reducing agent (Invitrogen) and RIPA 1X. Samples were then subjected to SDS-PAGE and transferred onto PVDF membranes (Millipore). Membranes were activated by absolute ethanol followed by electro-transfer and saturation with PBS supplemented with $0.1 \%$ Tween 20 (Sigma-Aldrich) and 5\% nonfat milk. Membranes were incubated with primary antibody, washed three times in PBS supplemented with $0.1 \%$ Tween 20 (Sigma-Aldrich), incubated with mouse (NA931; GE Healthcare) or rabbit (NA934; GE Healthcare) secondary antibody coupled to peroxidase, washed three times in PBS supplemented with $0.1 \%$ Tween 20 (Sigma-Aldrich), and revealed by chemiluminescence with ECL Western blotting detection system (GE Healthcare). Images were acquired with the PXi4 GeneSys software version 1.3.9.0.

RNA isolation and RT-qPCR. Total RNA was extracted using the RNeasy minikit (QIAGEN). First-strand cDNA was synthesized using the TaqMan RNA-to- $\mathrm{C}_{\mathrm{T}} 1$ Step kit (Life Technologies) and RT-qPCR was performed using the ABI 7500 fast real-time PCR system (Applied Biosystems) and the Taqman RT-PCR mix (Applied Biosystems), with primers pik3r1. Data obtained from the RT-qPCR reaction was analyzed using the comparative threshold cycle $(\mathrm{Ct})$ method with GAPDH used as the reference gene for normalization.

Intragastric oral inoculation. Mice were infected by gavage with $10^{9}$ CFUs of bacteria in $300 \mu \mathrm{l}$ of PBS containing $50 \mathrm{mg} / \mathrm{mL}$ of $\mathrm{CaCO}_{3}$. After $5 \mathrm{~h}$, the intestinal jejunum or ileum loops were taken.

Ligated loop assay. Before intestinal surgery, animals were fasted overnight to reduce their gastrointestinal contents. Animals were anesthetized by injecting $100 \mu \mathrm{l}$ of $100 \mathrm{mg} / \mathrm{kg}$ ketamine and $8 \mathrm{mg} / \mathrm{kg}$ xylazine in PBS. Laparotomy incision was made to expose the cecum and the small intestine. Two experiments were designed. In the first experiment, the intestine was ligated between the medial jejunum and distal ileum, isolating a loop of $\sim 23 \mathrm{~cm}$ of small intestine. $10^{9} \mathrm{CFUs}$ of $\mathrm{Lm}$ in $700 \mu \mathrm{l}$ of sterile PBS were slowly injected into the loop with a 30-gauge needle. After $30 \mathrm{~min}$, all Peyer's patches and 
$2 \mathrm{~cm}$ of jejunum or ileum without Peyer's patches were excised from the loop. The tissues were incubated for $2 \mathrm{~h}$ in DMEM supplemented with Glutamax (DMEM + Glutamax) containing $100 \mu \mathrm{g} / \mathrm{ml}$ gentamicin to kill extracellular bacteria, then washed in sterile PBS and weighed. Tissues were then homogenized in sterile PBS and plated on BHI agar plates. In the second experiment, $2-3 \mathrm{~cm}$ were isolated from the ileal part of the intestine and $100 \mu \mathrm{l}$ of wortmannin $(10 \mu \mathrm{M})$ diluted in sterile PBS was injected with a 30 -gauge needle. After $1 \mathrm{~h}$, tissues were removed, washed three times in DMEM + Glutamax, and fixed overnight in $4 \%$ paraformaldehyde in PBS for $2 \mathrm{~h}$ at room temperature, and then embedded in $4 \%$ low-melting-point agarose (Sigma-Aldrich) for $200-\mu \mathrm{M}$-thick vibratome sections.

Microscopy. Samples were imaged with an Axio-observer (Carl Zeiss) or with an LSM700 confocal microscope (Carl Zeiss) with $40 \times$ water immersion objective or a $40 \times$ oil-immersion objective. Wavelengths of the lasers used on the LSM 700 were 405, 488, 555, and $639 \mathrm{~nm}$. Image capture and analysis were performed using AxioVision (Carl Zeiss) and Zen software (Carl Zeiss).

Immunoblotting and confocal imaging quantification assays. Immunoblotting quantifications were calculated with Genetools software (Syngene) on membranes pictures taken with PXi4 GeneSys software version 1.3.9.0.

Imaging quantifications were made on confocal microscopy pictures. The different cell subsets were identified with ECCD2 or HECD-1 antibodies (enterocytes), NKM 16-2-4 antibody (M cells), or WGA (goblet cells) combined with a nuclear Hoechst staining. For each cell subset, P-Akt intensity was quantified by measuring the mean intensity of pixels with the ICY software. P-Akt intensity quantifications were done on at least 10 fields from 2 independent experiments (intestinal vibratome sections, placental explants sections, or cells on coverslips) for each tested conditions.

Statistical analysis. Statistical comparisons were made using the unpaired Student's $t$ test, the Mann-Whitney $U$ test, the one-way ANOVA or the two-way ANOVA test followed by a Bonferroni's multiple comparisons test as indicated. $\mathrm{P}$ values $<0.05$ were considered significant. Significant differences are marked as follows: *, $\mathrm{P}<0.05 ; * *, \mathrm{P}<0.01$; ***, $\mathrm{P}<0.001$; $* * * *, \mathrm{P}<0.0001$.

We thank the members of the Biology of Infection Unit for their help and support. Grégoire Gessain is supported by an AXA PhD fellowship, and Yu-Huan Tsai by a Pasteur-Paris University International PhD Program fellowship funded by Institut Carnot. We thank Brice Sperandio and Philippe Sansonetti for providing the LS174T cell line. We thank Dr. Laurent Salomon and Ms. Anne-Isabelle Boulogne for providing human placental tissue samples. We thank the PIRC at Institut Pasteur for assistance with regulatory and ethical guidelines.

The Biology of Infection Unit is supported by Institut Pasteur, Institut National de la Santé et de la Recherche Médicale, Fondation pour la Recherche Médicale, Ville de Paris, Fondation BNP Paribas, Listress FP7 program, the LabEx IBEID, and the European Research Council.

The authors have no conflicting financial interests.

Submitted: 25 July 2014

Accepted: 7 January 2015

\section{REFERENCES}

Bécavin, C., C. Bouchier, P. Lechat, C. Archambaud, S. Creno, E. Gouin, Z. Wu, A. Kühbacher, S. Brisse, M.G. Pucciarelli, et al. 2014. Comparison of widely used Listeria monocytogenes strains EGD, 10403S, and EGD-e highlights genomic variations underlying differences in pathogenicity. MBio. 5:e00969-e14. http://dx.doi.org/10.1128/mBio.00969-14

Biggs, W.H. III, J. Meisenhelder, T. Hunter, W.K. Cavenee, and K.C. Arden. 1999. Protein kinase B/Akt-mediated phosphorylation promotes nuclear exclusion of the winged helix transcription factor FKHR1. Proc. Natl. Acad. Sci. USA. 96:7421-7426. http://dx.doi.org/10.1073/pnas.96 .13 .7421
Braun, L., B. Ghebrehiwet, and P. Cossart. 2000. gC1q-R/p32, a C1qbinding protein, is a receptor for the InlB invasion protein of Listeria monocytogenes. EMBO J. 19:1458-1466. http://dx.doi.org/10.1093/ emboj/19.7.1458

Bucior, I., J.F. Pielage, and J.N. Engel. 2012. Pseudomonas aeruginosa pili and flagella mediate distinct binding and signaling events at the apical and basolateral surface of airway epithelium. PLoS Pathog. 8:e1002616. http://dx.doi.org/10.1371/journal.ppat.1002616

Chiba, S., T. Nagai, T. Hayashi, Y. Baba, S. Nagai, and S. Koyasu. 2011. Listerial invasion protein internalin B promotes entry into ileal Peyer's patches in vivo. Microbiol. Immunol. 55:123-129. http://dx.doi.org/10.1111/j $.1348-0421.2010 .00292 . x$

Cossart, P., J. Pizarro-Cerdá, and M. Lecuit. 2003. Invasion of mammalian cells by Listeria monocytogenes: functional mimicry to subvert cellular functions. Trends Cell Biol. 13:23-31. http://dx.doi.org/10.1016/ S0962-8924(02)00006-5

Cummings, H.E., J. Barbi, P. Reville, S. Oghumu, N. Zorko, A. Sarkar, T.L. Keiser, B. Lu, T. Rückle, S. Varikuti, et al. 2012. Critical role for phosphoinositide 3-kinase gamma in parasite invasion and disease progression of cutaneous leishmaniasis. Proc. Natl. Acad. Sci. USA. 109:12511256. http://dx.doi.org/10.1073/pnas.1110339109

Disson, O., and M. Lecuit. 2013. In vitro and in vivo models to study human listeriosis: mind the gap. Microbes Infect. 15:971-980. http://dx.doi.org/ 10.1016/j.micinf.2013.09.012

Disson, O., S. Grayo, E. Huillet, G. Nikitas, F. Langa-Vives, O. Dussurget, M. Ragon, A. Le Monnier, C. Babinet, P. Cossart, and M. Lecuit. 2008. Conjugated action of two species-specific invasion proteins for fetoplacental listeriosis. Nature. 455:1114-1118. http://dx.doi.org/10.1038/ nature 07303

Disson, O., G. Nikitas, S. Grayo, O. Dussurget, P. Cossart, and M. Lecuit. 2009. Modeling human listeriosis in natural and genetically engineered animals. Nat. Protoc. 4:799-810. http://dx.doi.org/10.1038/nprot.2009.66

Doran, K.S., A. Banerjee, O. Disson, and M. Lecuit. 2013. Concepts and mechanisms: crossing host barriers. Cold Spring Harb Perspect Med. 3:1. http://dx.doi.org/10.1101/cshperspect.a010090

Dramsi, S., I. Biswas, E. Maguin, L. Braun, P. Mastroeni, and P. Cossart. 1995. Entry of Listeria monocytogenes into hepatocytes requires expression of inIB, a surface protein of the internalin multigene family. Mol. Microbiol. 16:251-261. http://dx.doi.org/10.1111/j.1365-2958.1995. tb02297.x

Dramsi, S., S. Lévi, A. Triller, and P. Cossart. 1998. Entry of Listeria monocytogenes into neurons occurs by cell-to-cell spread: an in vitro study. Infect. Immun. 66:4461-4468.

Durual, S., C. Blanchard, M. Estienne, M.F. Jacquier, J.C. Cuber, V. Perrot, C. Laboisse, and J.C. Cuber. 2005. Expression of human TFF3 in relation to growth of HT-29 cell subpopulations: involvement of PI3-K but not STAT6. Differentiation. 73:36-44. http://dx.doi.org/10.1111/ j.1432-0436.2005.07301006.x

Engelman, J.A., J. Luo, and L.C. Cantley. 2006. The evolution of phosphatidylinositol 3-kinases as regulators of growth and metabolism. Nat. Rev. Genet. 7:606-619. http://dx.doi.org/10.1038/nrg1879

Gaillard, J.L., P. Berche, C. Frehel, E. Gouin, and P. Cossart. 1991. Entry of L. monocytogenes into cells is mediated by internalin, a repeat protein reminiscent of surface antigens from gram-positive cocci. Cell. 65:11271141. http://dx.doi.org/10.1016/0092-8674(91)90009-N

Girard, D., A. Leclercq, E. Laurent, M. Lecuit, H. de Valk, and V. Goulet. 2014. Pregnancy-related listeriosis in France, 1984 to 2011, with a focus on 606 cases from 1999 to 2011. Euro Surveill. 19:19.

Guo, J.P., D. Coppola, and J.Q. Cheng. 2011. IKBKE protein activates Akt independent of phosphatidylinositol 3-kinase/PDK1/mTORC2 and the pleckstrin homology domain to sustain malignant transformation. J. Biol. Chem. 286:37389-37398. http://dx.doi.org/10.1074/jbc .M111.287433

Hull, B.E., and L.A. Staehelin. 1976. Functional significance of the variations in the geometrical organization of tight junction networks. J. Cell Biol. 68:688-704. http://dx.doi.org/10.1083/jcb.68.3.688

Ireton, K., B. Payrastre, H. Chap, W. Ogawa, H. Sakaue, M. Kasuga, and P. Cossart. 1996. A role for phosphoinositide 3-kinase in bacterial 
invasion. Science. 274:780-782. http://dx.doi.org/10.1126/science.274 .5288 .780

Ireton, K., B. Payrastre, and P. Cossart. 1999. The Listeria monocytogenes protein InlB is an agonist of mammalian phosphoinositide 3-kinase. J. Biol. Chem. 274:17025-17032. http://dx.doi.org/10.1074/jbc.274.24.17025

Jacquet, C., M. Doumith, J.I. Gordon, P.M. Martin, P. Cossart, and M. Lecuit. 2004. A molecular marker for evaluating the pathogenic potential of foodborne Listeria monocytogenes. J. Infect. Dis. 189:2094-2100. http://dx.doi.org/10.1086/420853

Jang, M.H., M.N. Kweon, K. Iwatani, M. Yamamoto, K. Terahara, C. Sasakawa, T. Suzuki, T. Nochi, Y. Yokota, P.D. Rennert, et al. 2004. Intestinal villous $\mathrm{M}$ cells: an antigen entry site in the mucosal epithelium. Proc. Natl. Acad. Sci. USA. 101:6110-6115. http://dx.doi.org/10.1073/ pnas.0400969101

Jiwani, S., Y. Wang, G.C. Dowd, A. Gianfelice, P. Pichestapong, B. Gavicherla, N. Vanbennekom, and K. Ireton. 2012. Identification of components of the host type IA phosphoinositide 3-kinase pathway that promote internalization of Listeria monocytogenes. Infect. Immun. 80:12521266. http://dx.doi.org/10.1128/IAI.06082-11

Jonquières, R., J. Pizarro-Cerdá, and P. Cossart. 2001. Synergy between the $\mathrm{N}$ - and C-terminal domains of InlB for efficient invasion of nonphagocytic cells by Listeria monocytogenes. Mol. Microbiol. 42:955-965. http://dx.doi.org/10.1046/j.1365-2958.2001.02704.x

Khelef, N., M. Lecuit, H. Bierne, and P. Cossart. 2006. Species specificity of the Listeria monocytogenes InlB protein. Cell. Microbiol. 8:457-470. http://dx.doi.org/10.1111/j.1462-5822.2005.00634.x

Kölsch, V., T. Seher, G.J. Fernandez-Ballester, L. Serrano, and M. Leptin. 2007. Control of Drosophila gastrulation by apical localization of adherens junctions and RhoGEF2. Science. 315:384-386. http://dx.doi.org/10 $.1126 /$ science. 1134833

Kourtis, A.P., J.S. Read, and D.J. Jamieson. 2014. Pregnancy and infection. N. Engl.J. Med. 370:2211-2218. http://dx.doi.org/10.1056/NEJMra1213566

Lecuit, M. 2007. Human listeriosis and animal models. Microbes Infect. 9:12161225. http://dx.doi.org/10.1016/j.micinf.2007.05.009

Lecuit, M., H. Ohayon, L. Braun, J. Mengaud, and P. Cossart. 1997. Internalin of Listeria monocytogenes with an intact leucine-rich repeat region is sufficient to promote internalization. Infect. Immun. 65:5309-5319.

Lecuit, M., S. Dramsi, C. Gottardi, M. Fedor-Chaiken, B. Gumbiner, and P. Cossart. 1999. A single amino acid in E-cadherin responsible for host specificity towards the human pathogen Listeria monocytogenes. EMBO J. 18:3956-3963. http://dx.doi.org/10.1093/emboj/18.14.3956

Lecuit, M., S. Vandormael-Pournin, J. Lefort, M. Huerre, P. Gounon, C. Dupuy, C. Babinet, and P. Cossart. 2001. A transgenic model for listeriosis: role of internalin in crossing the intestinal barrier. Science. 292:1722-1725. http://dx.doi.org/10.1126/science.1059852

Lecuit, M., D.M. Nelson, S.D. Smith, H. Khun, M. Huerre, M.C. VacherLavenu, J.I. Gordon, and P. Cossart. 2004. Targeting and crossing of the human maternofetal barrier by Listeria monocytogenes: role of internalin interaction with trophoblast E-cadherin. Proc. Natl. Acad. Sci. USA. 101:6152-6157. http://dx.doi.org/10.1073/pnas.0401434101

Madara, J.L., and J.S. Trier. 1982. Structure and permeability of goblet cell tight junctions in rat small intestine. J. Membr. Biol. 66:145-157. http:// dx.doi.org/10.1007/BF01868490

Madara, J.L., J.S. Trier, and M.R. Neutra. 1980. Structural changes in the plasma membrane accompanying differentiation of epithelial cells in human and monkey small intestine. Gastroenterology. 78:963-975.

Maehama, T., and J.E. Dixon. 1998. The tumor suppressor, PTEN/MMAC1, dephosphorylates the lipid second messenger, phosphatidylinositol 3,4,5trisphosphate. J. Biol. Chem. 273:13375-13378. http://dx.doi.org/10 $.1074 / \mathrm{jbc} .273 .22 .13375$

Mahajan, K., and N.P. Mahajan. 2012. PI3K-independent AKT activation in cancers: a treasure trove for novel therapeutics. J. Cell. Physiol. 227:31783184. http://dx.doi.org/10.1002/jcp.24065

Marchiando, A.M., L. Shen, W.V. Graham, K.L. Edelblum, C.A. Duckworth, Y. Guan, M.H. Montrose, J.R. Turner, and A.J. Watson. 2011. The epithelial barrier is maintained by in vivo tight junction expansion during pathologic intestinal epithelial shedding. Gastroenterology. 140:1208-1218. http://dx.doi.org/10.1053/j.gastro.2011.01.004
McDole, J.R., L.W. Wheeler, K.G. McDonald, B. Wang, V. Konjufca, K.A. Knoop, R.D. Newberry, and M.J. Miller. 2012. Goblet cells deliver luminal antigen to CD103+ dendritic cells in the small intestine. Nature. 483:345-349. http://dx.doi.org/10.1038/nature10863

McGuckin, M.A., S.K. Lindén, P. Sutton, and T.H. Florin. 2011. Mucin dynamics and enteric pathogens. Nat. Rev. Microbiol. 9:265-278. http:// dx.doi.org/10.1038/nrmicro2538

Mengaud, J., H. Ohayon, P. Gounon, P. Cossart, and P. CossartMege R-M. 1996. E-cadherin is the receptor for internalin, a surface protein required for entry of L. monocytogenes into epithelial cells. Cell. 84:923932. http://dx.doi.org/10.1016/S0092-8674(00)81070-3

Neutra, M.R., A. Frey, and J.P. Kraehenbuhl. 1996. Epithelial M cells: gateways for mucosal infection and immunization. Cell. 86:345-348. http://dx.doi.org/10.1016/S0092-8674(00)80106-3

Nikitas, G., C. Deschamps, O. Disson, T. Niault, P. Cossart, and M. Lecuit. 2011. Transcytosis of Listeria monocytogenes across the intestinal barrier upon specific targeting of goblet cell accessible E-cadherin. J. Exp. Med. 208:2263-2277. http://dx.doi.org/10.1084/jem .20110560

Pattillo, R.A., G.O. Gey, E. Delfs, W.Y. Huang, L. Hause, D.J. Garancis, M. Knoth, J. Amatruda, J. Bertino, H.G. Friesen, and R.F. Mattingly. 1971. The hormone-synthesizing trophoblastic cell in vitro: a model for cancer research and placental hormone synthesis. Ann. N. Y. Acad. Sci. 172(10 Physiology a):288-298. http://dx.doi.org/10.1111/j.17496632.1971.tb34942.x

Pentecost, M., J. Kumaran, P. Ghosh, and M.R. Amieva. 2010. Listeria monocytogenes internalin $\mathrm{B}$ activates junctional endocytosis to accelerate intestinal invasion. PLoS Pathog. 6:e1000900. http://dx.doi.org/10 .1371/journal.ppat.1000900

Perez, T.D., M. Tamada, M.P. Sheetz, and W.J. Nelson. 2008. Immediateearly signaling induced by E-cadherin engagement and adhesion. J. Biol. Chem. 283:5014-5022. http://dx.doi.org/10.1074/jbc.M705209200

Phan, Q.T., C.L. Myers, Y. Fu, D.C. Sheppard, M.R. Yeaman, W.H. Welch, A.S. Ibrahim, J.E. Edwards Jr., and S.G. Filler. 2007. Als3 is a Candida albicans invasin that binds to cadherins and induces endocytosis by host cells. PLoS Biol. 5:e64. http://dx.doi.org/10.1371/journal. pbio.0050064

Porvaznik, M., W. Baker, and R.I. Walker. 1983. Disruption of the goblet cell intercellular junction following histamine infusion of the rabbit ileum. Experientia. 39:514-518. http://dx.doi.org/10.1007/BF01965183

Robbins, J.R., and A.I. Bakardjiev. 2012. Pathogens and the placental fortress. Curr. Opin. Microbiol. 15:36-43. http://dx.doi.org/10.1016/j.mib .2011.11.006

Robbins, J.R., K.M. Skrzypczynska, V.B. Zeldovich, M. Kapidzic, and A.I. Bakardjiev. 2010. Placental syncytiotrophoblast constitutes a major barrier to vertical transmission of Listeria monocytogenes. PLoS Pathog. 6:e1000732. http://dx.doi.org/10.1371/journal.ppat.1000732

Saeed, M.F., A.A. Kolokoltsov, A.N. Freiberg, M.R. Holbrook, and R.A. Davey. 2008. Phosphoinositide-3 kinase-Akt pathway controls cellular entry of Ebola virus. PLoS Pathog. 4:e1000141. http://dx.doi.org/10 .1371 journal.ppat.1000141

Shen, Y., M. Naujokas, M. Park, and K. Ireton. 2000. InIB-dependent internalization of Listeria is mediated by the Met receptor tyrosine kinase. Cell. 103:501-510. http://dx.doi.org/10.1016/S0092-8674(00)00141-0

Swaminathan, B., and P. Gerner-Smidt. 2007. The epidemiology of human listeriosis. Microbes Infect. 9:1236-1243. http://dx.doi.org/10.1016/j micinf.2007.05.011

Tom, B.H., L.P. Rutzky, M.M. Jakstys, R. Oyasu, C.I. Kaye, and B.D. Kahan. 1976. Human colonic adenocarcinoma cells. I. Establishment and description of a new line. In Vitro. 12:180-191. http://dx.doi.org/ 10.1007/BF02796440

Tsai, Y.H., O. Disson, H. Bierne, and M. Lecuit. 2013. Murinization of internalin extends its receptor repertoire, altering Listeria monocytogenes cell tropism and host responses. PLoS Pathog. 9:e1003381. http://dx.doi .org/10.1371/journal.ppat.1003381

Vanhaesebroeck, B., L. Stephens, and P. Hawkins. 2012. PI3K signalling: the path to discovery and understanding. Nat. Rev. Mol. Cell Biol. 13:195203. http://dx.doi.org/10.1038/nrm3290 
Wang, M.L., S.A. Keilbaugh, T. Cash-Mason, X.C. He, L. Li, and G.D. Wu. 2008. Immune-mediated signaling in intestinal goblet cells via PI3-kinase- and AKT-dependent pathways. Am. J. Physiol. Gastrointest. Liver Physiol. 295:G1122-G1130. http://dx.doi.org/10.1152/ajpgi.90430.2008

Wollert, T., B. Pasche, M. Rochon, S. Deppenmeier, J. van den Heuvel, A.D. Gruber, D.W. Heinz, A. Lengeling, and W.-D. Schubert. 2007.
Extending the host range of Listeria monocytogenes by rational protein design. Cell. 129:891-902. http://dx.doi.org/10.1016/j.cell.2007.03.049

Yusta, B., D. Holland, J.A. Koehler, M. Maziarz, J.L. Estall, R. Higgins, and D.J. Drucker. 2009. ErbB signaling is required for the proliferative actions of GLP-2 in the murine gut. Gastroenterology. 137:986-996. http://dx.doi.org/10.1053/j.gastro.2009.05.057 